\title{
An In-Situ Diagnostic Study of Electromagnetic Stirring Effects on Peritectic Solidification Kinetics for Containerlessly Processed Liquid Fe-Ti Alloys
}

\begin{abstract}
Y.H. WU, J. CHANG, L. HU, S. SHA, X. CAI, S.S. XU, and B. WEI
The electromagnetic stirring (EMS) effects on peritectic solidification kinetics of undercooled liquid Fe-Ti alloys have been investigated by electrostatic levitation (ESL) and electromagnetic levitation (EML) methods assisted with in-situ diagnostic techniques. The high-sensitivity pyrometer and high-speed camera were employed to monitor the complete solidification process for levitated liquid $\mathrm{Fe}_{59} \mathrm{Ti}_{41}$ alloy in undercooling $\Delta T$ range of $0 \mathrm{~K}$ to $213 \mathrm{~K}$. Theoretical calculations showed that there existed EMS inside electromagnetically levitated alloy melts, and the internal fluid flow dynamics depended on levitation height and melt undercooling. As $\Delta T$ rised, the primary dendrite growth velocity $V$ increased according to a power function. Meanwhile, the peritectic recalescence degree $\Delta T_{\mathrm{pr}}$ and the peritectic recalescence rate $R_{\mathrm{pr}}$ were enhanced gradually, whereas the peritectic recalescence time $t_{\mathrm{pr}}$ and the peritectic solidification time $t_{\mathrm{ps}}$ were shortened linearly. The comparison between ESL and EML experiments revealed that the EMS resulted in four respects of influences including (1) dendrite growth effect, (2) concentration field effect, (3) peritectic reaction effect and (4) microstructure evolution effect. In contrast with ESL, the $V$ of $\mathrm{Fe}_{50} \mathrm{Ti}_{50}$ alloy measured by EML was slightly larger at small undercoolings, indicating the EMS affected dendrite growth processes. The solute concentration $C_{\mathrm{L}}^{*}$ around primary $\mathrm{Fe}_{2} \mathrm{Ti}$ dendrites for electrostatically levitated liquid $\mathrm{Fe}_{59} \mathrm{Ti}_{41}$ alloy deviated far away from original composition, while the EMS homogenized concentration field and the $C_{\mathrm{L}}^{*}$ variation was weak under EML condition. Both $t_{\mathrm{pr}}$ and $t_{\mathrm{ps}}$ in the absence of EMS were longer that those in the presence of EMS, and it was demonstrated that the EMS accelerated peritectic reaction. Except for microstructure refinement, the EMS modulated the microstructure type and also changed the faceted-growth mode of intermetallic compound phases.
\end{abstract}

https://doi.org/10.1007/s11661-020-05745-w

(c) The Minerals, Metals \& Materials Society and ASM International 2020

\section{INTRODUCTION}

A lot of technologically important materials, such as iron-based alloys, titanium-based alloys, copper-based alloys, nickel-based alloys and aluminum-based alloys, belong to peritectic alloy systems. ${ }^{[1-6]}$ In the process of melt cooling, these peritectic alloys usually exhibit typical peritectic reaction, i.e., primary solid phase $(\alpha)$ reacts with the remaining liquid $(L)$ to form a second solid phase $(\beta) \cdot{ }^{[5,6]}$ The properties of peritectic alloys are closely related to the corresponding solidification processes. ${ }^{[7-9]}$ A fundamental understanding of peritectic solidification kinetics is highly desirable because it plays an important role in the technological advances. Owing

Y. H. WU, J. CHANG, L. HU, S. SHA, X. CAI, S. S. XU, and B. WEI are with the Department of Applied Physics, Northwestern Polytechnical University, No. 127 of West Youyi Road, Xi'an, 710072, Shannxi Province, P.R. China. Contact e-mail: bbwei@nwpu.edu.cn Manuscript submitted September 21, 2019.

Article published online April 9, 2020 to scientific interests and practical applications, peritectic solidification behavior has been an active research topic in the past decade. ${ }^{[10-14]}$ Furthermore, extensive experimental and theoretical investigations have been performed to understand solidification behaviors of peritectic alloys. ${ }^{[12-17]}$ Nevertheless, most of previous investigations mainly focused on the solidification mechanism selections, microstructure characteristics modulations and mechanical property optimizations. So far, the attention to peritectic solidification kinetics is rather limited especially at extremely non-equilibrium solidification conditions due to the experimental difficulty in conducting an in-situ observation. ${ }^{[18]}$ Thus, much work still needs to be done to get a better understanding of peritectic solidification kinetics.

Fortunately, with the help of undercooling techniques, ${ }^{[18-23]}$ liquid peritectic alloys achieve non-equilibrium solidification in a relatively slow cooling process. This makes it possible to investigate the peritectic solidification process when the in-situ diagnostic techniques are employed simultaneously. In recent years, using containerless processing techniques of electrostatic 
levitation (ESL), ${ }^{[18-20]}$ electromagnetic levitation (EML), ${ }^{[21,22]}$ aerodynamic levitation ${ }^{[23]}$ and acoustic levitation $^{[24]}$ assisted with a high-speed photodiode and a high-speed camera, the undercooling dependence of primary dendrite growth velocity has been measured for various peritectic alloys, and the primary dendrite growth kinetics can be determined accordingly. ${ }^{[14,19]}$ As it is very difficult to obtain the substantial undercoolings, primary dendrite growth kinetics in supercooled states has scarcely been reported. As we all known, the peritectic solidification process involves not only the primary dendrite growth but also the subsequent peritectic reaction. At present, conducting an in-situ study for the measurements of peritectic reaction time and peritectic reaction intensity is still difficult in undercooled state. Up to now, little attentions have been paid to clarify peritectic reaction kinetics in experiments. Furthermore, the competition between primary dendrite growth and subsequent peritectic reaction is not understood completely. Although many phase-field models $^{[15,17]}$ have recently been proposed to simulate primary dendrite growth and subsequent peritectic reaction processes, most of these models cannot be used to analyze peritectic solidification kinetics in highly undercooled state. Therefore, it is urgent to explore the kinetics of primary dendrite growth and subsequent peritectic reaction for undercooled peritectic-type alloy melts.

Electromagnetic stirring (EMS) method is a good candidate to homogenize solute elements and refine solidification microstructures so as to improve the alloy properties. ${ }^{[25-31]}$ As the EMS is introduced into the alloy solidification processes, it considerably accelerates the mass/heat transfer within metallic melts. Typical peritectic solidification process can be divided into three stages: (1) nucleation and growth of primary phase from undercooled alloy melts; (2) peritectic reaction of the primary phase with the remaining liquid phase; (3) the produced peritectic solid phase around primary solid phase suppresses the proceeding of peritectic reaction, and the remnant liquid phase directly solidifies during further cooling. It can be believed that the EMS influences the dynamic process of peritectic solidification to some extent. Currently, no open literature reports the EMS effects on the peritectic solidification kinetics of undercooled alloy melts, and the corresponding underlying mechanisms still remain elusive. More importantly, the quantitative relationships between the peritectic solidification kinetics and melt undercooling have never been involved in the previous literatures. To contribute to the advancement of peritectic solidification theory, it is necessary to carry out an in-situ diagnostic study of EMS effects on peritectic solidification kinetics for undercooled peritectic alloy melts.

Among various undercooling techniques, both the electrostatic levitation (ESL) and the electromagnetic levitation (EML) are frequently used to achieve the high undercooling and rapid solidification of liquid alloys in slow cooling processes. ${ }^{[19]}$ During the levitation experiments, with the help of a high-speed camera, a high-precision pyrometer and a high-sensitivity photodiode, the peritectic solidification kinetics of levitated alloy melts can be in-situ determined quantitatively in ESL and EML experiments. In contrast with the ESL processing process, there exists intensive electromagnetic stirring (EMS) within the electromagnetically levitated alloy melts. Besides, fluid flow dynamics induced by the EMS could be analyzed by those physical models in References 32 through 34. If we further compare the determined peritectic solidification kinetics under the ESL and EML conditions, the EMS effects on the peritectic solidification kinetics and rapid solidification characteristics will be experimentally demonstrated. Consequently, performing a comparative study of peritectic solidification kinetics between ESL and EML experiments is of great importance for an in-depth understanding of EMS effects.

Figure 1 displays the selected alloy compositions in Fe-Ti phase diagram. As the EMS is absent from the electrostatically levitated hypoperitectic $\mathrm{Fe}_{59} \mathrm{Ti}_{41}$ alloy, the solute composition $C_{\mathrm{L}}^{*}$ around growing primary $\mathrm{Fe}_{2} \mathrm{Ti}$ dendrites deviates greatly to $\mathrm{FeTi}$ phase side. In this case, $C_{\mathrm{L}}^{*}$ maybe falls in the composition range of metastable coupled growth between primary and peritectic phases, ${ }^{[14]}$ and the solidification microstructures perhaps show some differences in comparison to those at EML state. Besides, the hyperperitectic $\mathrm{Fe}_{50} \mathrm{Ti}_{50}$ alloy is also chosen to explore the effects of solidification temperature interval on the solidification behavior selections as well as the EMS influences on microstructure evolutions. Taking the $\mathrm{Fe}_{59} \mathrm{Ti}_{41}$ and $\mathrm{Fe}_{50} \mathrm{Ti}_{50}$ alloys as examples, the aim of this work is to investigate the EMS effects on peritectic solidification kinetics in undercooled state through a comparative ESL and EML experiments assisted with various in-situ diagnostic techniques. During the peritectic solidification, the EMS plays a crucial role in the dendrite growth, solute redistribution, peritectic reaction and microstructure evolution, and the four kinds of effects will be clarified one by one in the following sections.

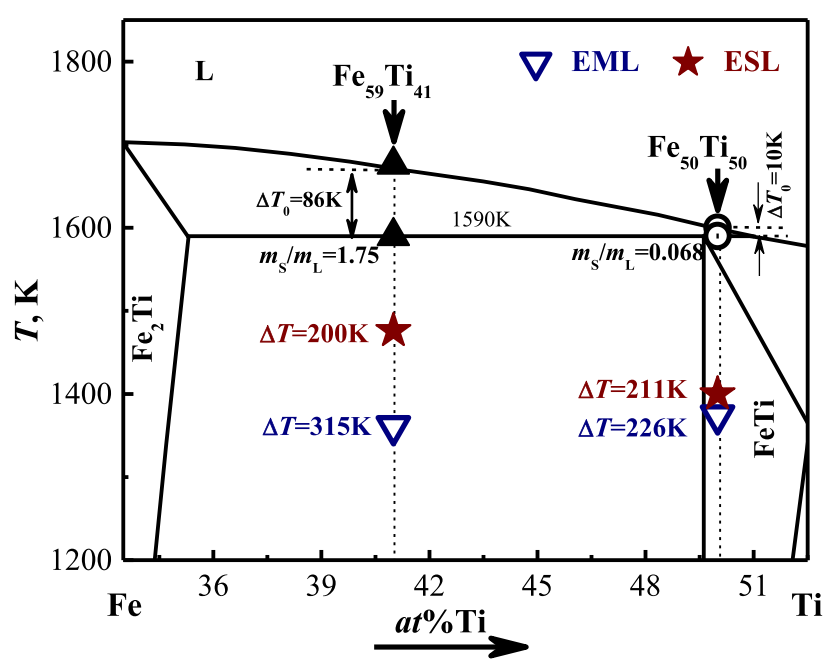

Fig. 1-Selected alloy composition selections and achieved maximum undercoolings during electrostatic levitation (ESL) and electromagnetic levitation (EML) experiments. 


\section{EXPERIMENTAL PROCEDURE}

\section{A. Preparation of Master Alloys}

The master alloys with hypoperitectic $\mathrm{Fe}_{59} \mathrm{Ti}_{41}$ and hyperperitectic $\mathrm{Fe}_{50} \mathrm{Ti}_{50}$ compositions for electrostatic levitation (ESL) and electromagnetic levitation (EML) experiments were made from high purity elements of $\mathrm{Fe}$ (99.999 pct) and Ti (99.999 pct). Before the EML processing, the experimental samples were synthesized inside an ultrahigh vacuum arc-melting facility, whereas the alloy samples in ESL experiment were prepared by laser melting in an inert gas atmosphere. To satisfy experimental requirements, the sample mass could be adjusted so that it would produce spheres of about 3 and $6 \mathrm{~mm}$ in diameters for ESL and EML experiments, respectively.

\section{B. Electrostatic Levitation (ESL) Experiments}

During ESL experiments in Figure 2, small alloy spheres with $\mathrm{Fe}_{59} \mathrm{Ti}_{41}$ and $\mathrm{Fe}_{50} \mathrm{Ti}_{50}$ compositions were levitated in an ultrahigh vacuum $\left(\sim 10^{-5} \mathrm{~Pa}\right)$ environment within an alternating electrostatic field. Using a continuous wave SPISP300 fiber laser with $1070 \mathrm{~nm}$ wavelength, the electrostatically levitated sample was continually heated until it was completely melted and overheated for several seconds. Afterwards, the levitated alloy melt was naturally cooled by stopping laser irradiation, during which the sample temperature was detected by a CellaTemp PA 40 single-color pyrometer with an absolutely accuracy of $\pm 5 \mathrm{~K}$, which was calibrated using the liquidus temperature of levitated samples. A series of melting and cooling cycles were repeated to access desirable undercoolings. The in-situ diagnosis of two successive recalescences on the cooling curve corresponding to primary dendrite growth and subsequent peritectic reaction was conducted with the aid of a high-sensitivity PDA100A-EC photoelectric detector and a Redlake HG 100K high-speed camera. These recalescences images in $256 \times 192$ pixels were recorded at a frame rate of 2000 frames per second (fps).

\section{Electromagnetic Levitation (EML) Experiments}

During EML experiments in Figure 3(a), binary $\mathrm{Fe}_{59} \mathrm{Ti}_{41}$ and $\mathrm{Fe}_{50} \mathrm{Ti}_{50}$ alloys were levitated and heated by $\mathrm{RF}$ induction furnace in a high vacuum chamber $\left(\sim 10^{-5} \mathrm{~Pa}\right)$ refilled with Ar protecting gas to $\sim 10^{-5} \mathrm{~Pa}$. When the alloy sample was completely melted and overheated above its liquidus temperature for tens of seconds, the levitated molten alloy was subsequently cooled by the forced cooling He gas around the sample surface. As the melt temperature was decreased below its liquidus temperature, the liquid alloy started to locate at an undercooled state and spontaneous nucleation phenomena perhaps occurred at some undercoolings. Here, the melting and cooling processes were repeated until the desirable undercooling was obtained. In the process of the EML processing, the temperature signal of levitated alloys was in-situ monitored by an infrared pyrometer of CellaTemp PA20 with an absolutely accuracy of $\pm 5 \mathrm{~K}$, and meanwhile the rapid solidification processes were recorded by a PDA100A-EC photoelectric detector and a Redlake HG 100K high-speed camera. Those recalescences images in $512 \times 384$ pixels were recorded at a frame rate of 2000 frames per second (fps).

\section{Solidification Microstructure Characterization}

After the $\mathrm{Fe}_{59} \mathrm{Ti}_{41}$ and $\mathrm{Fe}_{50} \mathrm{Ti}_{50}$ alloys were processed using the ESL and EML methods, these undercooled alloy samples were then mounted and polished before etching. A solution composed of $1 \mathrm{~mL} \mathrm{HF}+2.5 \mathrm{~mL}$ $\mathrm{HNO}_{3}+1.5 \mathrm{~mL} \mathrm{HCl}+95 \mathrm{~mL} \mathrm{H}_{2} \mathrm{O}$ was used to etch those experimental samples. The rapid solidification microstructures were characterized by an FEI Sirion 200 scanning electron microscope (SEM) equipped with an INCA 300 energy dispersive X-ray spectroscope (EDS), a Zeiss Axiovert 200 MAT optical microscope and a X-ray computed tomography (XCT) using a phoenix nanotom m CT system (GE Sensing \& Inspection Technologies), and the final phase constitutions were identified by a Rigaku D/max 2500 X-ray diffractometer.

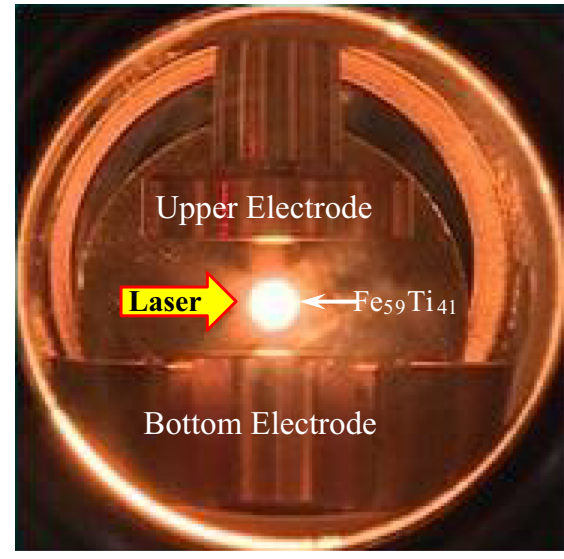

(a)

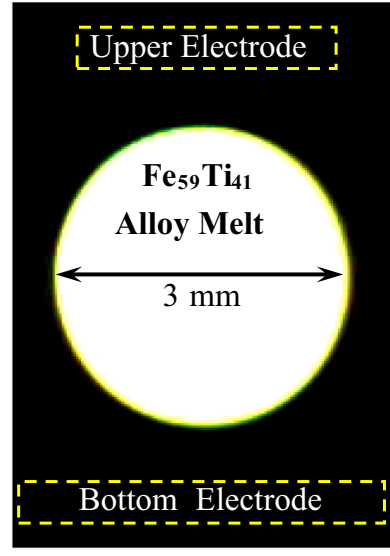

(b)

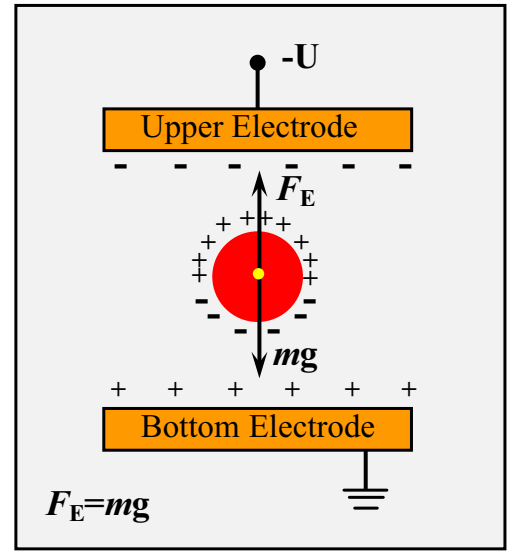

(c)

Fig. 2-Electrostatic levitation experiments of liquid $\mathrm{Fe}_{59} \mathrm{Ti}_{41}$ alloy: $(a)$ a zoom shot of levitating process, $(b)$ enlarged view of a levitated sample, (c) a schematic model. 


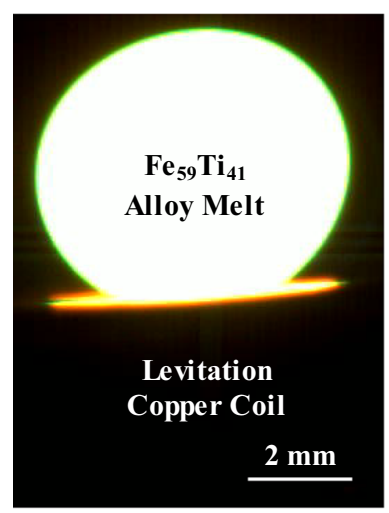

(a)

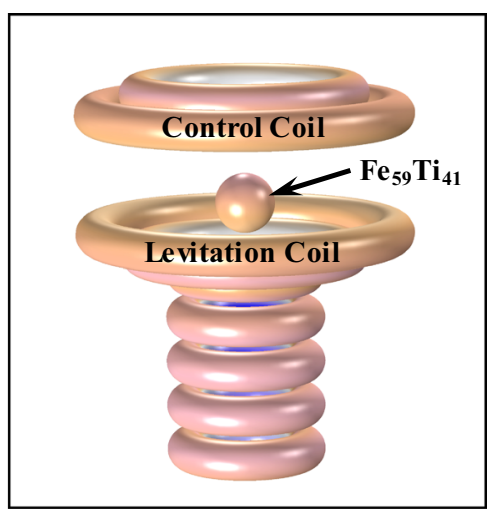

(b)

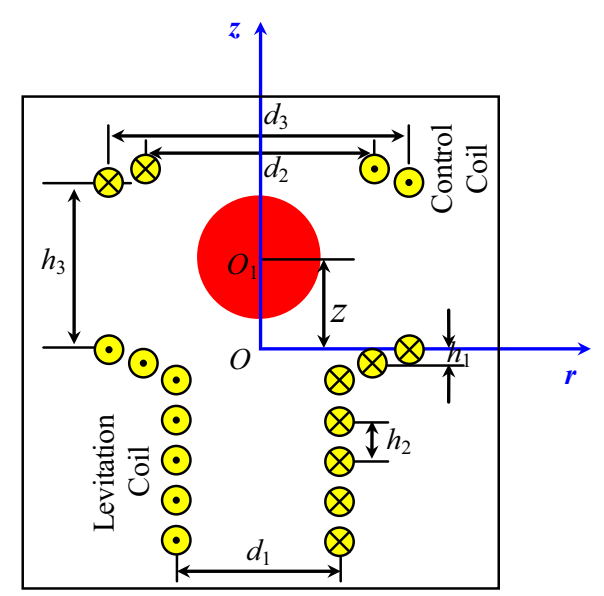

(c)

Fig. 3-A computational model of fluid flow dynamics within electromagnetically levitated $\mathrm{Fe}_{59} \mathrm{Ti}_{41}$ alloy melt: $(a)$ a snapshot of levitated liquid sample, (b) levitation coil configuration, $(c)$ a schematic 2D model.

\section{RESULTS AND DISCUSSION}

\section{A. Theoretical Prediction of Electromagnetic Stirring Effects}

1. Governing equations for fluid flow dynamics within electromagnetically levitated alloy melts

Figure 3(a) provides the photograph of electromagnetically levitated $\mathrm{Fe}_{59} \mathrm{Ti}_{41}$ alloy melt. The coil configuration used in EML experiments is illustrated in Figure 3(b). Evidently, this copper coil possesses nine turns, in which the main winding with seven turns at the configuration bottom mainly produces the levitation force to suspend the sample, and the winding with two turns at the top is responsible for the levitation stability. To analyze the fluid flow dynamics induced by the electromagnetic stirring inside electromagnetically levitated $\mathrm{Fe}_{59} \mathrm{Ti}_{41}$ drops, a two-dimensional computational model is given in Figure 3(c). In order to simplify the calculation process, the levitated $\mathrm{Fe}_{59} \mathrm{Ti}_{41}$ drop is supposed to keep a spherical shape with $6 \mathrm{~mm}$ diameter, and no rotation phenomenon takes place during the levitation. As the temperature difference inside the levitated small droplet is smaller than 1 $\mathrm{K},{ }^{[35]}$ the Marangoni convection caused by the temperature gradient could be ignored, and special attentions are paid to the forced convection induced by the EMS. Before calculating the fluid flow dynamics, it needs to firstly compute the electromagnetic field in the coil configuration by the time-harmonic Maxwell equations:

$$
\begin{gathered}
\nabla \times \boldsymbol{H}=\boldsymbol{J}+\varepsilon_{0} \frac{\partial \boldsymbol{E}}{\partial t} \\
\nabla \times \boldsymbol{E}=-\frac{\partial \boldsymbol{B}}{\partial t} \\
\nabla \cdot \boldsymbol{B}=0
\end{gathered}
$$

$$
\boldsymbol{J}=\sigma \boldsymbol{E}
$$

$$
F=0.5 \operatorname{Re}(\boldsymbol{J} \times \boldsymbol{B})
$$

where $\boldsymbol{H}$ is the magnetic field intensity, $\boldsymbol{J}$ is the induced current intensity, $\boldsymbol{E}$ is the electric field intensity, $\varepsilon_{0}$ is the electric permeability, $\boldsymbol{B}$ is the magnetic flux density, $\sigma$ is the electrical conductivity and $\boldsymbol{F}$ is the Lorenz force. As the Lorenz force equals the sample weight, the internal flow field can be obtained by solving the Reynolds averaged turbulence model $k-\varepsilon$ for an incompressible fluid. ${ }^{[35]}$ In that case, the Lorentz force acts as a source term in the Navier-Stokes equation and the mass conservation equation:

$$
\begin{gathered}
\frac{\partial \boldsymbol{V}}{\partial t}+(\boldsymbol{V} \cdot \nabla) \boldsymbol{V}=-\frac{1}{\rho} \nabla \cdot\left(p \boldsymbol{I}+\mu\left(\nabla \boldsymbol{V}+(\nabla \boldsymbol{V})^{T}\right)\right) \\
+\frac{1}{\rho} \boldsymbol{F}+g \\
\nabla \cdot \boldsymbol{V}=0 \\
\boldsymbol{V} \cdot \boldsymbol{n}=0
\end{gathered}
$$

where $\boldsymbol{V}$ is the fluid flow velocity, $\mu$ is the dynamic viscosity, $p$ is the pressure, $g$ is the gravity acceleration, $\boldsymbol{I}$ is the unit matrix, $\boldsymbol{n}$ is the unit normal vector, and the detailed descriptions about $k-\varepsilon$ model can be found elsewhere. ${ }^{[35,36]}$ The physical parameters of the coil configuration as well as the liquid $\mathrm{Fe}_{59} \mathrm{Ti}_{41}$ alloy are listed in Table I. Here, the liquid alloy parameters are estimated by the linear superposition of pure elements. ${ }^{[39]}$ 
Table I. Physical Parameters of Liquid $\mathrm{Fe}_{59} \mathrm{Ti}_{41}$ Alloy Used for Calculations

\begin{tabular}{|c|c|c|c|c|c|c|}
\hline \multirow[b]{2}{*}{ Physical Parameter } & \multicolumn{6}{|c|}{ Undercooling $\Delta T(\mathrm{~K})$} \\
\hline & 0 & 50 & 100 & 150 & 200 & 250 \\
\hline Density $\rho\left(\mathrm{g} / \mathrm{cm}^{3}\right)$ & 5.937 & 5.967 & 5.998 & 6.029 & 6.059 & 6.090 \\
\hline Viscosity $\eta(\mathrm{mPa} \cdot \mathrm{s})$ & 3.840 & 3.948 & 4.063 & 4.185 & 4.316 & 4.455 \\
\hline Surface Tension $\sigma,(\mathrm{N} / \mathrm{m})$ & 1.850 & 1.869 & 1.889 & 1.909 & 1.929 & 1.948 \\
\hline Thermal Conductivity $\lambda \mathrm{W} /(\mathrm{m} \cdot \mathrm{K})$ & 26.232 & 25.449 & 24.667 & 23.884 & 23.102 & 22.319 \\
\hline Electrical Conductivity $\sigma, 10^{6}(\mathrm{~S} / \mathrm{m})$ & 0.9191 & 1.0246 & 1.1574 & 1.3298 & 1.5625 & 1.8975 \\
\hline Coil Diameter $d_{1}(\mathrm{~mm})$ & 12 & 12 & 12 & 12 & 12 & 12 \\
\hline Coil Diameter $d_{2}(\mathrm{~mm})$ & 21 & 21 & 21 & 21 & 21 & 21 \\
\hline Coil Diameter $d_{3}(\mathrm{~mm})$ & 30 & 30 & 30 & 30 & 30 & 30 \\
\hline Coil Height $h_{1}(\mathrm{~mm})$ & 2 & 2 & 2 & 2 & 2 & 2 \\
\hline Coil Height $h_{2}(\mathrm{~mm})$ & 4.5 & 4.5 & 4.5 & 4.5 & 4.5 & 4.5 \\
\hline Coil Height $h_{3},(\mathrm{~mm})$ & 12 & 12 & 12 & 12 & 12 & 12 \\
\hline Current Frequency $f(\mathrm{kHz})$ & 200 & 200 & 200 & 200 & 200 & 200 \\
\hline
\end{tabular}

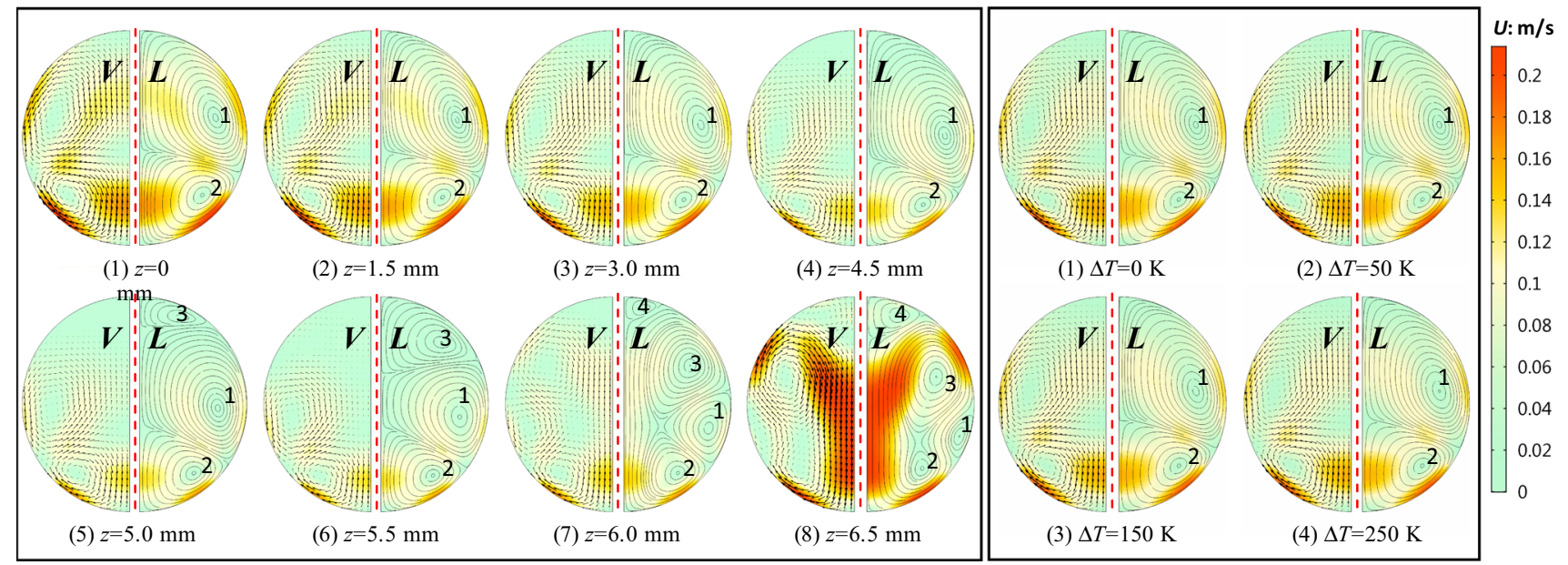

(a) $\Delta T=150 \mathrm{~K}$ : Levitation height $z$ effects

(b) $z=3 \mathrm{~mm}$ : Undercooling $\Delta T$ effects

Fig. 4-Effects of levitation height and bulk undercooling on fluid flow patterns within electromagnetically levitated $\mathrm{Fe}_{59} \mathrm{Ti}_{41}$ alloy melt: $(a)$ height effect, $(b)$ undercooling effect. Here, left and right semicircles denote velocity field $(V)$ and streamline field $(L)$, respectively.

2. Fluid flow patterns and velocity distribution spectra

By using the above model, the fluid flow patterns within the electromagnetically levitated $\mathrm{Fe}_{59} \mathrm{Ti}_{41}$ alloy melt were simulated as functions of levitation height and melt undercooling, and the results are depicted in Figure 4. Here, the left and right semicircles denote the velocity field and streamline field, respectively. It can be seen clearly that the effect of levitation height on fluid flow patterns are more evident than the undercooling effect. In the undercooling range of $0 \mathrm{~K}$ to $250 \mathrm{~K}$, there are always two anti-circulating fluid vortices caused by electromagnetic stirring for each half of the levitated $\mathrm{Fe}_{59} \mathrm{Ti}_{41}$ drop. The undercooling effect on the fluid flow pattern is so weak that it can be ignored. In the two-dimensional case, the fluid vortex \#1 is clockwise while the fluid vortex \#2 displays an anticlockwise direction.
In EML experiments, the larger the alternating current on the copper coil, the higher the sample levitation position. Before the melt cooling, the sample levitation position is relatively low and it makes the sample obtain a relatively high superheating. In the process of the melt cooling, the sample levitation height is greatly enhanced by increasing the alternating current, and this contributes to achieving a relatively large cooling rate for levitated droplets. As shown in Figure 4(a), the fluid flow patterns strongly depend on the levitation height $z$. In the small $z$ regime below 4.5 $\mathrm{mm}$, a clockwise fluid vortex \#1 and an anticlockwise fluid vortex \#2 are observed within each half of the levitated droplet. Moreover, with the rise in the $z$, the vortex centers of \#1 and \#2 slightly deviate to the earth ground. Meanwhile, the fluid vortex \#1 gradually becomes larger and the vortex \#2 dwindles. Once the 
levitation height increases above $4.5 \mathrm{~mm}$, three fluid vortices start to occur for each half of the levitated droplet, as illustrated in Figures 4(a5) and (a6). In these cases, the fluid vortex \#1 still possesses a clockwise direction, whereas fluid vortices \#2 and \#3 are anticlockwise. When the levitation height further rises to 6 and $6.5 \mathrm{~mm}$, four fluid vortices are produced for each half of the levitated droplet, as shown in Figures 4(a7) and (a8). Moreover, the neighboring vortices are anti-circulating. It can be concluded that there exists intensive forced convection induced by the electromagnetic stirring within the electromagnetically levitated $\mathrm{Fe}_{59} \mathrm{Ti}_{41}$ alloy melt, and the internal fluid flow patterns are mainly dominated by the levitation height.

Figure 5 shows the calculated internal fluid flow velocities of electromagnetically levitated $\mathrm{Fe}_{59} \mathrm{Ti}_{41}$ alloy melt as functions of bulk undercooling $\Delta T$ and levitation height $z$. The maximum fluid velocity $U_{\mathrm{Max}}$ and the average fluid velocity $U_{\text {Ave }}$ are closely related to the levitation height and bulk undercooling. In the $z$ range of 0 to $6.5 \mathrm{~mm}$, the maximum fluid velocity $U_{\text {Max }}$ attains $21.4 \mathrm{~cm} / \mathrm{s}$ in the calculated undercooling range, whereas the average fluid velocity $U_{\text {Ave }}$ reaches $9.8 \mathrm{~cm} / \mathrm{s}$. The $U_{\text {Max }}$ and $U_{\text {Ave }}$ monotonously decrease as the undercooling is enhanced. This is easy to be understood by referring to the melt viscosity. In highly undercooled state, the rising viscosity leads to the decline in the fluid flow velocity. In the small $z$ regime below $5.5 \mathrm{~mm}$, both the $U_{\text {Max }}$ and $U_{\text {Ave }}$ present a decreasing trend with the enlarged $z$. Once the $z$ rises to $5.5 \mathrm{~mm}$, both the $U_{\mathrm{Max}}$ and $U_{\text {Ave }}$ attain smallest values. As the $z$ further increases beyond this threshold value, these two velocities begin to increase. The evolution of fluid flow pattern and the appearance of minimum fluid velocity with the $z$ variation can be ascribed to competitive effects between electromagnetic fields in the upper winding and lower winding of the levitation coil.

Figure 6 depicts the distribution spectra of fluid flow velocity inside electromagnetically levitated $\mathrm{Fe}_{59} \mathrm{Ti}_{41}$ alloy melt. When the levitation height is $0 \mathrm{~mm}$, the internal fluid flow velocity is not homogeneous and the peak value locates at about $8 \mathrm{~cm} / \mathrm{s}$, as illustrated in
Figures 6(a1) and (a4). Through increasing the alternating current, the levitation height rises to $5 \mathrm{~mm}$, the fluid velocity field still presents non-homogeneous characteristics. In contrast with the previous one, the peak value of velocity distribution spectra becomes smaller and it approaches $6.1 \mathrm{~cm} / \mathrm{s}$, as observed in Figure 6(a4). As the levitation height further increases to $6.5 \mathrm{~mm}$, the fluid velocity field is relatively homogeneous, as seen in Figures 6(a3) and (a4). This indicates that the sample levitation height plays a crucial role in the distribution spectra of fluid flow velocity field for undercooled melts under EML conditions. Besides, the effect of bulk undercooling on the distribution characteristics of fluid flow velocity is also explored as shown in Figure 6(b). Evidently, it can be found that bulk undercooling has little effect on the distribution spectra.

\section{Analyses of EMS Effects on Peritectic Solidification}

As mentioned, the electromagnetic stirring (EMS) results in the appearance of forced convection inside the electromagnetically levitated alloy melt. The EMS influences on the typical peritectic solidification process are theoretically analyzed and they can be classified into four aspects.

a. Dendrite growth effect Extensive investigations ${ }^{[37,38]}$ of convection effects on the dendrite growth demonstrated that the dendrite growth is enhanced on the upstream side and suppressed on the downstream side. Furthermore, the growth velocity of dendrite tip will reduce if the angle between preferred growth direction of the upstream direction and a dendrite arm varies from 0 to $180 \mathrm{deg}$. During the spontaneous nucleation and growth of primary phase for undercooled Fe-Ti liquid alloys in EML experiments, the growth direction of those dendrites is random and the forced convection in the levitated drop is very complicated. Although it is difficult to predict the acceleration degree of the dendrite growth, the growth velocity measurements under the influences of EMS have a high possibility to be affected as compared with those in the absence of EMS.

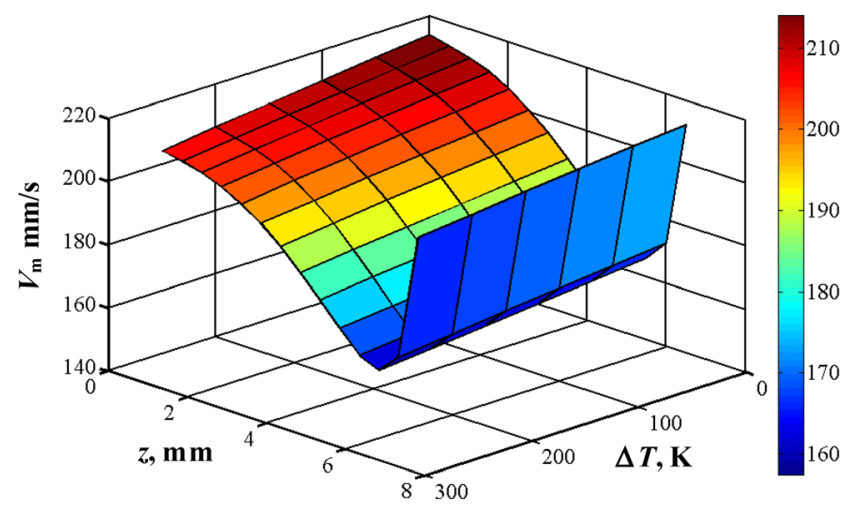

(a) $V_{\mathrm{m}}-\Delta T-z$

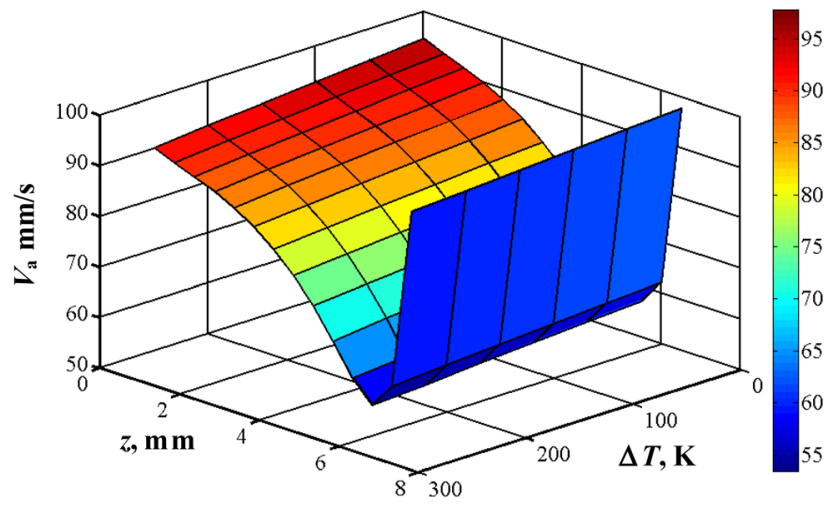

(b) $V_{\mathrm{a}}-\Delta T-\mathrm{z}$

Fig. 5-Internal fluid flow velocities of electromagnetically levitated $\mathrm{Fe}_{59} \mathrm{Ti}_{41}$ alloy melt $v$ s levitation height $z$ and bulk undercooling $\Delta T$ : (a) maximum fluid velocity $V_{\mathrm{m}},(b)$ average fluid velocity $V_{\mathrm{a}}$. 


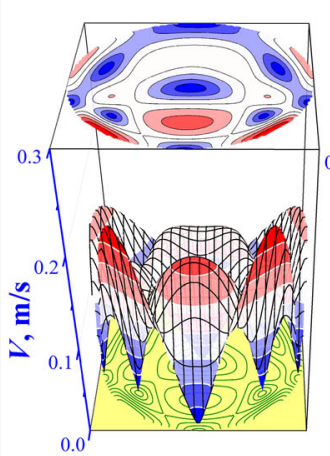

(a1) $z=0 \mathrm{~mm}$

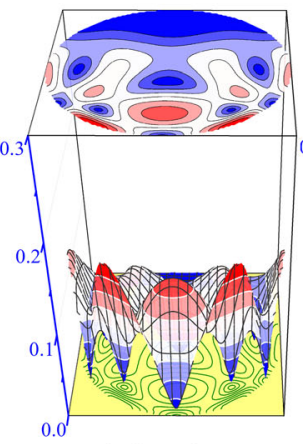

(a2) $z=5 \mathrm{~mm}$

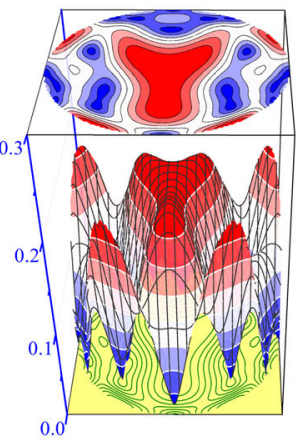

(a3) $z=6.5 \mathrm{~mm}$

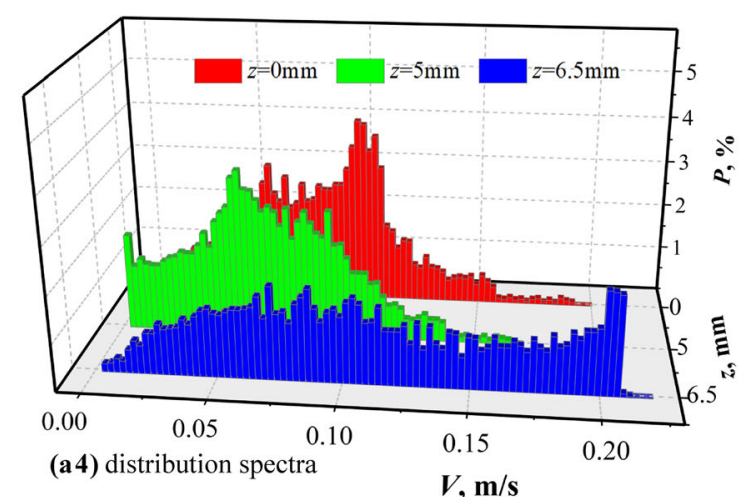

$\boldsymbol{V}, \mathbf{m} / \mathbf{s}$

(a) Levitation height $z$ effects at $\Delta T=150 \mathrm{~K}$

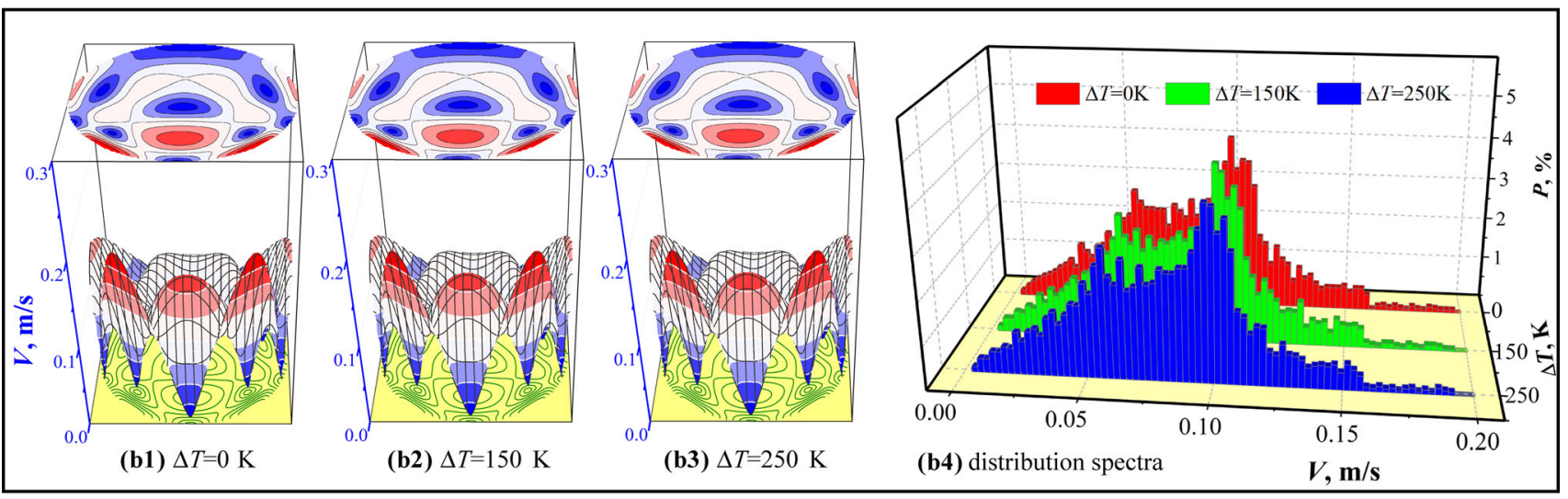

(b) Bulk undercooling $\Delta T$ effects at $z=3 \mathrm{~mm}$

Fig. 6-Effects of levitation height and bulk undercooling on the distribution spectra of fluid flow velocity within electromagnetically levitated liquid $\mathrm{Fe}_{59} \mathrm{Ti}_{41}$ alloy: (a) levitation height $z$ effects at $\Delta T=150 \mathrm{~K},(b)$ bulk undercooling $\Delta T$ effects at $z=3.0 \mathrm{~mm}$.

b. Concentration field effect As primary $\mathrm{Fe}_{2} \mathrm{Ti}$ phase nucleates and grows from the undercooled $\mathrm{Fe}_{59} \mathrm{Ti}_{41}$ liquid alloy, the solute concentration $C_{\mathrm{L}}^{*}$ at the front of liquid/solid interface considerably deviates away from the original concentration in the absence of EMS. Because the EMS greatly facilitates the mass transfer and homogenizes the solute element, the $C_{\mathrm{L}}^{*}$ around those $\mathrm{Fe}_{2} \mathrm{Ti}$ dendrites is close to the original composition if the EMS is imposed. Obviously, the concentration field in the presence of EMS is more homogeneous than that without EMS effects. The concentration field difference maybe leads to the distinctions of solidification mechanisms and microstructure characteristics.

c. Peritectic reaction effect The peritectic reaction of $L$ $+\alpha \rightarrow \beta$ is usually sluggish. If EMS is introduced into the metallic melts, primary solid phase is considerably refined as a result of grain fragmentation and multiplication. Later, the refined solid phase reacts more quickly with the remaining liquid owing to the enhancement of specific surface area for the contact between solid grains and accelerating melt flow. In addition, peritectic reaction is an atom diffusion process, and the solute diffusion acceleration induced by EMS contributes to the proceeding of peritectic reaction. d. Microstructure evolution effect It has been widely accepted that the forced convection caused by EMS improves the fragmentation and thus facilitates grain multiplication to form refined microstructures. As analyzed above, the concentration field difference between the cases of EMS existence and EMS absence perhaps results in the distinction of microstructure type. In this work, the solidification microstructures are always composed of intermetallic compound phases, which are possible to grow in the faceted-growth mode. Considering the EMS induces the mechanical collision and fluid erosion between growing grains and remaining melt, the faceted-growth feature of the compound phase may be changed due to the fluid erosion and fluid scouring. This disappearing process of the facetedgrowth morphology is similar with stone weathering.

\section{B. Experimental Confirmation of Electromagnetic Stirring Influences on Peritectic Solidification Kinetics}

1. Typical cooling processes of electrostatically and electromagnetically levitated $\mathrm{Fe}$-Ti alloy melts

Hypoperitectic $\mathrm{Fe}_{59} \mathrm{Ti}_{41}$ and hyperperitectic $\mathrm{Fe}_{50} \mathrm{Ti}_{50}$ alloys have been substantially undercooled using the ESL and EML methods. Liquid $\mathrm{Fe}_{59} \mathrm{Ti}_{41}$ alloy was respectively undercooled up to $200 \mathrm{~K}$ and $315 \mathrm{~K}$ at ESL 


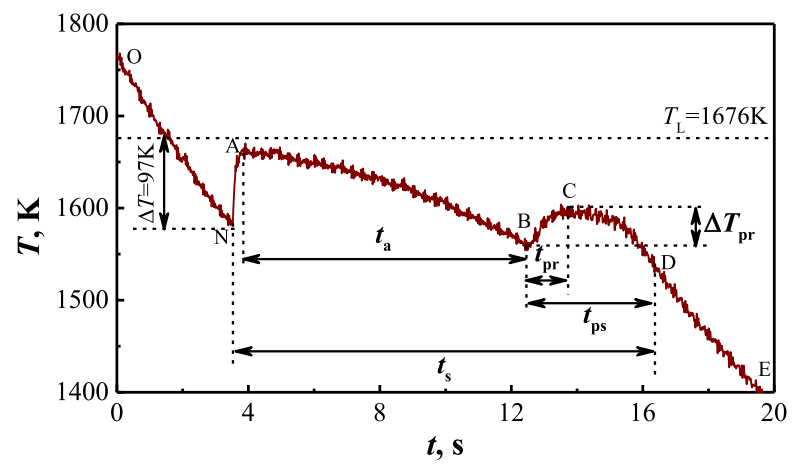

(a) Cooling curve at ESL state

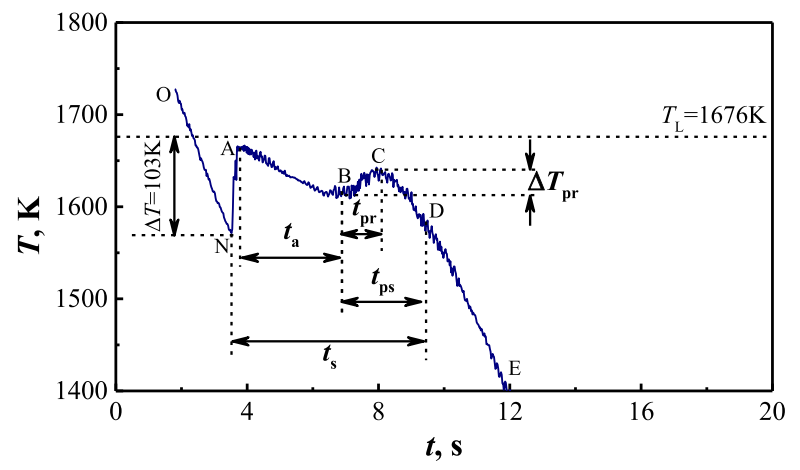

(b) Cooling curve at EML state

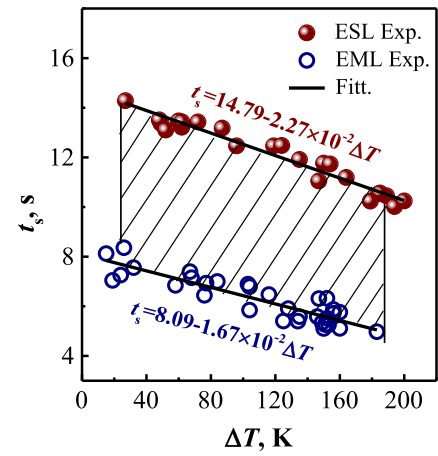

(c) $t_{\mathrm{s}}$

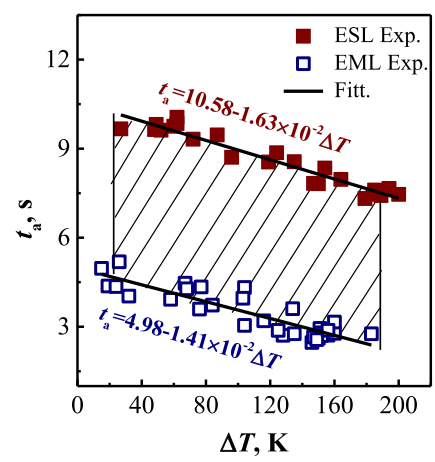

(d) $t_{\mathrm{a}}$

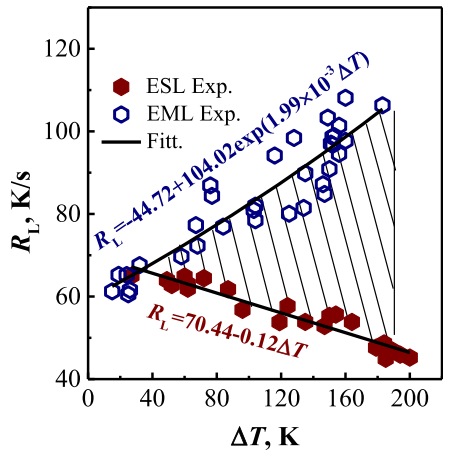

(e) $R_{\mathrm{L}}$

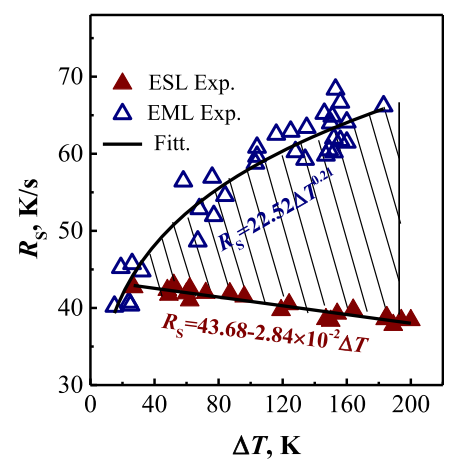

(f) $R_{\mathrm{S}}$

Fig. 7-A comparison of typical cooling curves between electrostatic levitation (ESL) and electromagnetic levitation (EML) experiments of $\mathrm{Fe}_{59} \mathrm{Ti}_{41}$ alloy: (a) cooling curve at ESL state, $(b)$ cooling curve at EML state, $(c)$ essential solidification time $t_{\mathrm{s}}$, $(d)$ primary thermal arrest time $t_{\mathrm{a}},(e)$ initial cooling rate $R_{\mathrm{L}}$ of liquid alloy before first recalescence, $(f)$ secondary cooling rate $R_{\mathrm{S}}$ of solidifying alloy after second recalescence.

and EML, whereas the $\mathrm{Fe}_{50} \mathrm{Ti}_{50}$ alloy obtained the maximum undercoolings of $211 \mathrm{~K}$ and $226 \mathrm{~K}$ in these two experiments. Taking the $\mathrm{Fe}_{59} \mathrm{Ti}_{41}$ alloy as an example, Figure 7 shows a characteristic comparison of typical cooling curves between ESL and EML experiments. It can be obviously seen that there are two exothermic peaks on the cooling curve in the experimental undercooling ranges of ESL and EML. The first one represents the rapid growth of primary $\mathrm{Fe}_{2} \mathrm{Ti}$ phase, whereas the second one denotes the subsequent formation of peritectic FeTi phase.

Although both the cooling curves under the two conditions possess the peritectic solidification characteristics, the cooling curves still display some minor differences in the case of same undercoolings, as shown in Figures 7(a) and (b). To analyze the characteristic differences, the typical cooling curve is divided into five stages, i.e., $\mathrm{O} \rightarrow \mathrm{N}, \mathrm{N} \rightarrow \rightarrow \mathrm{A}, \mathrm{A} \rightarrow \mathrm{B}, \mathrm{B} \rightarrow \mathrm{D}$ and $\mathrm{D} \rightarrow$ E. The duration times of $\mathrm{N} \rightarrow \mathrm{D}$ and $\mathrm{A} \rightarrow \mathrm{B}$ are defined as the essential solidification time $t_{\mathrm{s}}$ and primary thermal arrest time $t_{\mathrm{a}}$, respectively. The variations of $t_{\mathrm{s}}$ and $t_{\mathrm{a}}$ with bulk undercooling $\Delta T$ were statistically measured and the results are illustrated in Figures 7(c) and (d). Evidently, with the rise in the undercooling, $t_{\mathrm{s}}$ and $t_{\mathrm{a}}$ shorten linearly, irrespective of the levitation condition. However, owing to the forced cooling, the average cooling rate during the cooling in EML is much higher than that at ESL. In addition, due to the additional electromagnetic stirring in EML, both $t_{\mathrm{s}}$ and $t_{\mathrm{a}}$ are smaller than those at ESL. The dependences of $t_{\mathrm{s}}$ and $t_{\mathrm{a}}$ on the undercooling $\Delta T$ are simulated by

$$
\begin{aligned}
& t_{\mathrm{s}}=14.79-2.27 \times 10^{-2} \Delta T \text { at ESL } \\
& t_{s}=8.09-1.67 \times 10^{-2} \Delta T \text { at EML } \\
& t_{a}=10.58-1.63 \times 10^{-2} \Delta T \text { at ESL } \\
& t_{a}=4.98-1.41 \times 10^{-2} \Delta T \text { at EML }
\end{aligned}
$$

The average cooling rates in the stage of $\mathrm{O} \rightarrow \mathrm{N}$ and $\mathrm{D} \rightarrow \mathrm{E}$ are respectively named as the initial cooling rate $R_{\mathrm{L}}$ of liquid alloy before first recalescence and the secondary cooling rate $R_{\mathrm{S}}$ of solidifying alloy after second recalescence. The undercooling dependences of $R_{\mathrm{L}}$ and $R_{\mathrm{S}}$ were experimentally determined and plotted in Figures 7(e) and (f). $R_{\mathrm{L}}$ and $R_{\mathrm{S}}$ of the electromagnetically levitated $\mathrm{Fe}_{59} \mathrm{Ti}_{41}$ drop display an upward trend as the undercooling is increased, and their relationships can be expressed as

$$
R_{L}=44.72+104.02 \exp \left(1.99 \times 10^{-3} \Delta T\right) \text { at EML }
$$

$$
R_{S}=22.52 \Delta T^{0.21} \text { at EML }
$$


Under the ESL condition, the relationship between $R_{\mathrm{L}}$ (or $R_{\mathrm{S}}$ ) and $\Delta T$ satisfies a linear function:

$$
\begin{gathered}
R_{L}=70.44-0.12 \Delta T \text { at ESL } \\
R_{S}=43.68-2.84 \times 10^{-2} \Delta T \text { at ESL }
\end{gathered}
$$

In that case, $R_{\mathrm{L}}$ and $R_{\mathrm{S}}$ decrease monotonously with the enlarged $\Delta T$, which is absolutely opposite in contrast with EML results. The sample cooling in EML experiments mainly depends on the heat convection and it often has larger cooling rate due to the forced cooling. This is the reason why larger $R_{\mathrm{L}}$ and $R_{\mathrm{S}}$ are obtained at the EML condition. The levitated sample transmits heat only through radiation in ESL, thus the temperature drop is relatively sluggish. In other word, achieving a higher undercooling in ESL needs a longer time and the average cooling rate decreases with the undercooling.

During the cooling, double recalescence processes of liquid $\mathrm{Fe}_{59} \mathrm{Ti}_{41}$ alloy at ESL and EML are always observed in the experimental undercooling range. Figure 8 shows the snapshots of double recalescence processes for levitated liquid $\mathrm{Fe}_{59} \mathrm{Ti}_{41}$ alloy. For the first recalescence, the light color denotes the solidified primary $\mathrm{Fe}_{2} \mathrm{Ti}$ phase, and the undercooled liquid appears as dark color. Apparently, the rapid growth of primary $\mathrm{Fe}_{2} \mathrm{Ti}$ phase is characterized by liquid/solid interface migration until the whole levitated drop becomes bright. With the further extension of the cooling time, a second recalescence caused by peritectic reaction and transformation occurs subsequently. In such a case, this recalescence displays some discrete bright zones inside the dark matrix. In fact, these bright zones are experiencing the peritectic reaction and transformation. Moreover, the peritectic reaction and transformation are very sluggish in comparison to primary phase growth. At the two levitation conditions, the double recalescence processes are similar in the cases of same undercoolings.

\section{Primary dendrite growth kinetics under ESL} and EML conditions

According to the Fe-Ti phase diagram in Figure 1, the cooling curve in Figure 7, the recalescence process in Figure 8 and the XRD patterns in Figure 10(a), it is confirmed that the primary phase of undercooled $\mathrm{Fe}_{59} \mathrm{Ti}_{41}$ alloy corresponds to the $\mathrm{Fe}_{2} \mathrm{Ti}$ phase. Nevertheless, since the solidification temperature interval of $\mathrm{Fe}_{50} \mathrm{Ti}_{50}$ alloy is merely $10 \mathrm{~K}$, it may be argued that the metastable peritectic FeTi phase directly nucleates from the undercooled alloy melt, and primary phase is not $\mathrm{Fe}_{2} \mathrm{Ti}$ phase any more. The XRD analyses in Figure 12(a) reveal that the undercooled $\mathrm{Fe}_{50} \mathrm{Ti}_{50}$ alloy is only composed of FeTi phase. Meanwhile, considering the peritectic reaction and transformation cannot completely proceed, it could be determined that the FeTi phase directly nucleates and grows from undercooled $\mathrm{Fe}_{50} \mathrm{Ti}_{50}$ alloy melt, even if the undercooling is $24 \mathrm{~K}$.

A high-speed camera was employed to measure primary dendrite growth velocities of $\mathrm{Fe}_{59} \mathrm{Ti}_{41}$ alloy in ESL and EML experiments, and the results are plotted in Figure 9(a). The velocity data are basically consistent under the two conditions. With the enhancement in the undercooling, the solidification driving force enhances gradually, and primary $\mathrm{Fe}_{2} \mathrm{Ti}$ dendrite growth velocity of $\mathrm{Fe}_{59} \mathrm{Ti}_{41}$ alloy displays a rising trend. The relationship between the primary $\mathrm{Fe}_{2} \mathrm{Ti}$ dendrite growth velocity $V$ and bulk undercooling $\Delta T$ is described by a power function

$$
V=3.56 \times 10^{-4} \Delta T^{2.09}
$$

Figure 9(b) plots the dendrite growth velocity of FeTi phase for electromagnetically levitated and electrostatically levitated $\mathrm{Fe}_{50} \mathrm{Ti}_{50}$ alloys. A larger growth velocity is observed at a greater undercooling owing to the

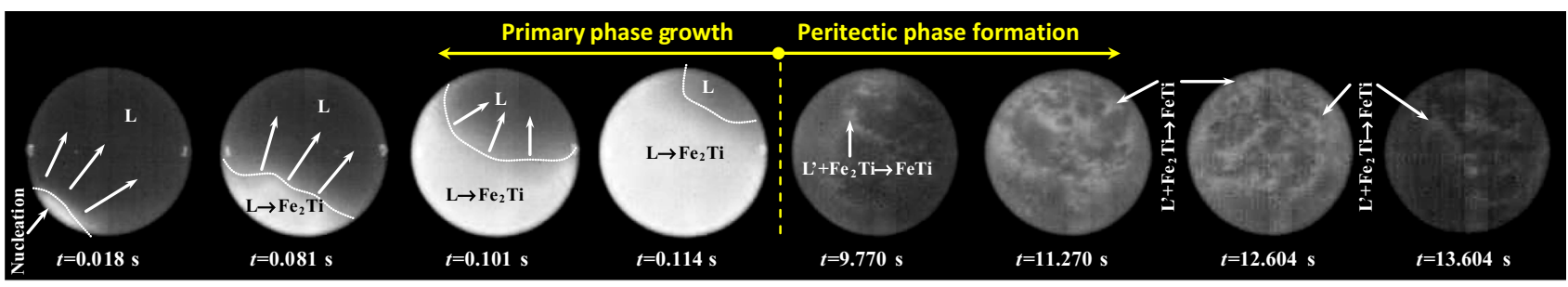

(a) $\mathrm{Fe}_{59} \mathrm{Ti}_{41}$ at ESL state

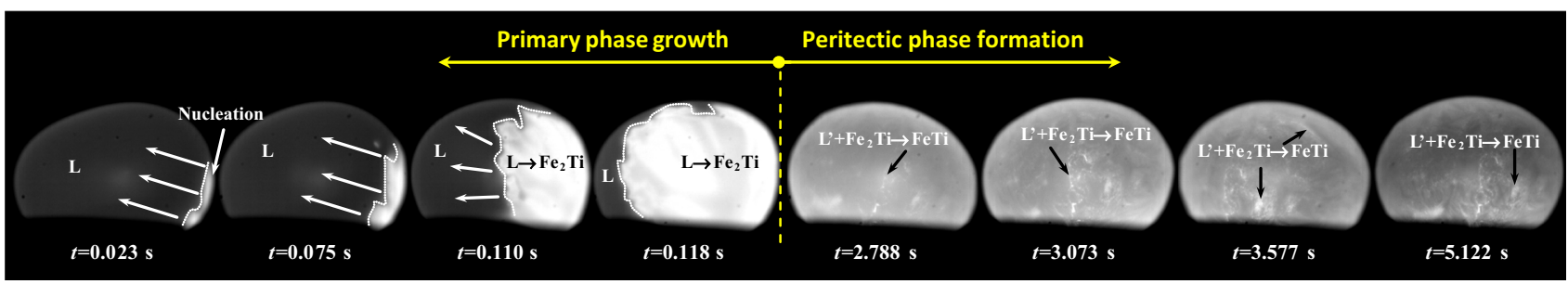

(b) $\mathrm{Fe}_{59} \mathrm{Ti}_{41}$ at EML state

Fig. 8-A high-speed photography observation of double recalescence processes for levitated liquid $\mathrm{Fe}_{59} \mathrm{Ti}_{41}$ alloy: $(a)$ electrostatically levitated $\mathrm{Fe}_{59} \mathrm{Ti}_{41}$ alloy subjected to $154 \mathrm{~K}$ undercooling, (b) electromagnetically levitated $\mathrm{Fe}_{59} \mathrm{Ti}_{41}$ alloy with $152 \mathrm{~K}$ undercooling. 


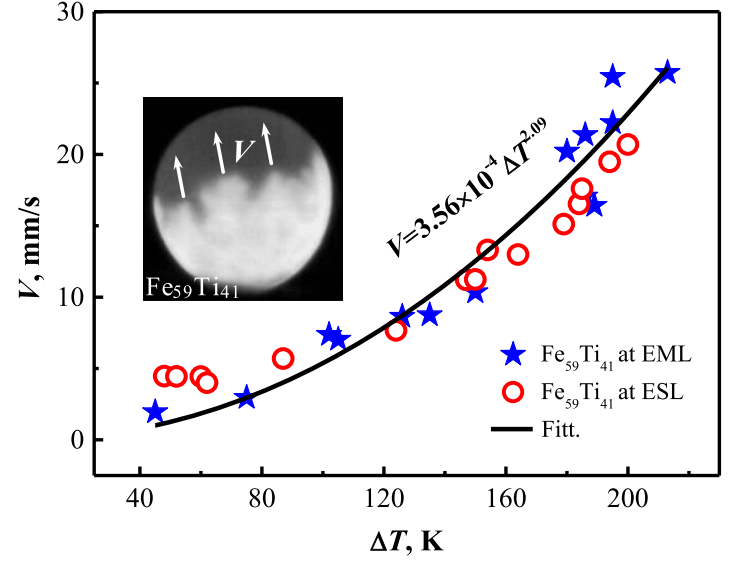

(a) $\mathrm{Fe}_{2} \mathrm{Ti}$ phase in $\mathrm{Fe}_{59} \mathrm{Ti}_{41}$ alloy

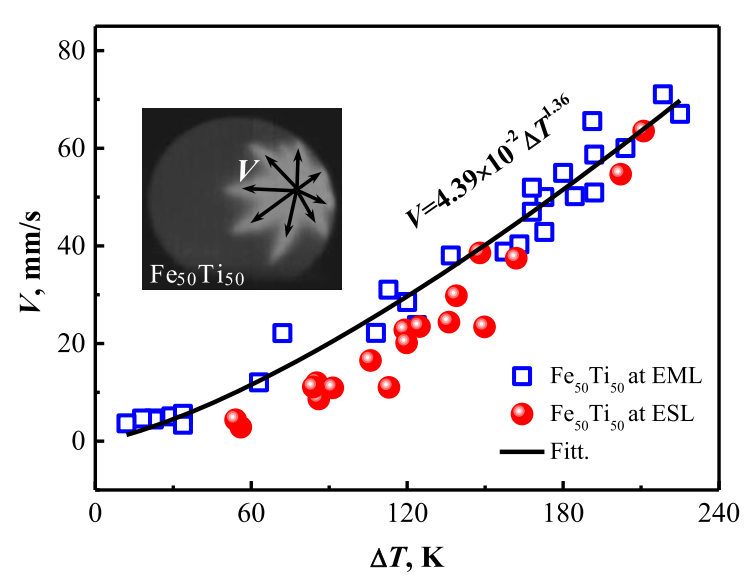

(b) FeTi phase of $\mathrm{Fe}_{50} \mathrm{Ti}_{50}$ alloy

Fig. 9-Dendrite growth velocity of undercooled Fe-Ti alloys: (a) $\mathrm{Fe}_{2} \mathrm{Ti}$ phase in liquid $\mathrm{Fe}_{59} \mathrm{Ti}_{41}$ alloy, (b) FeTi phase in liquid $\mathrm{Fe}_{50} \mathrm{Ti}_{50}$ alloy.

enhanced solidification driving force. The variation of FeTi dendrite growth velocity $V$ with bulk undercooling $\Delta T$ is represented by

$$
V=4.39 \times 10^{-2} \Delta T^{1.36}
$$

In the small undercooling regime below $150 \mathrm{~K}$, primary dendrite growth velocity measured by ESL is slightly lower than that determined by EML. Once the undercooling rises beyond this threshold value, the velocity differences between ESL and EML are gradually narrowing. Under slight undercoolings, the forced convection induced by the EMS is relatively strong. It greatly influences the velocity measurements, especially in upstream and downstream directions for those growing FeTi dendrites. However, these FeTi dendrites grow randomly within electromagnetically levitated $\mathrm{Fe}_{50} \mathrm{Ti}_{50}$ alloy melt, and there may be an angle between dendrite growth direction and forced convection direction. That perhaps leads to the slightly larger primary growth velocity at the EML condition. When the undercooling is improved, the forced convection is significantly weakened due to the drastic rise of melt viscosity. In that case, the EMS effect is too weak to affect the velocity measurement. This EMS effect cannot be reflected in the $V-\Delta T$ relation for $\mathrm{Fe}_{59} \mathrm{Ti}_{41}$ alloy, which may be attributed to two reasons. One is limited $V$ data in small undercooling range due to the difficulty in obtaining corresponding undercoolings, and the other is the relatively large systematic error because the $V$ is low.

3. Effects of electromagnetic stirring (EMS) on solute redistribution around growing primary dendrites

In contrast with electrostatically levitated alloy melts, there exist additional electromagnetic stirring (EMS) effects inside electromagnetically levitated alloy melts. The EMS plays a critical role in solute redistribution mechanisms around growing primary dendrites. During the nucleation and growth of primary $\mathrm{Fe}_{2} \mathrm{Ti}$ phase from the undercooled $\mathrm{Fe}_{59} \mathrm{Ti}_{41}$ alloy melt, iron and titanium elements respectively become depleted and enriched around growing $\mathrm{Fe}_{2} \mathrm{Ti}$ dendrites if we don't impose the
EMS. On the contrary, the EMS greatly accelerates the solute element transfer within undercooled alloy melts, and this contributes to homogenizing alloy melts even in the case of nucleation and growth of primary phase. As a result, local solute composition is close to the original alloy composition. Therefore, it can be speculated that the differences in the solute redistribution mechanisms result in microstructure characteristics distinctions between ESL and EML experiments.

To experimentally confirm this speculation, Figure 10 shows a microstructure comparison of $\mathrm{Fe}_{59} \mathrm{Ti}_{41}$ alloy under ESL and EML conditions, expecting to find the trace of EMS effects on solute redistribution behavior. The XRD analyses in Figure 10(a) revealed that undercooled $\mathrm{Fe}_{59} \mathrm{Ti}_{41}$ alloy consists of $\mathrm{Fe}_{2} \mathrm{Ti}$ and FeTi two intermetallic compound phases. Primary $\mathrm{Fe}_{2} \mathrm{Ti}$ and peritectic FeTi phases appear bright and dark, respectively. In the case of $213 \mathrm{~K}$ undercooling, the electromagnetically levitated $\mathrm{Fe}_{59} \mathrm{Ti}_{41}$ alloy forms a typical peritectic solidification microstructure, as seen in Figures $10(\mathrm{c} 1)$ and (c2). As for an almost equal undercooling at ESL condition, a hypoeutectic-like (or hyperperitectic-like) microstructure is observed in Figures 10(b1) and (b2). A little of eutectic-like microstructures are distributed inside interdendritic regions. In addition, it is also found that the rapid solidification microstructure processed by EML is refiner than that subjected to ESL.

The characteristic difference of solidification microstructures between the electrostatically and electromagnetically levitated $\mathrm{Fe}_{59} \mathrm{Ti}_{41}$ alloys can be interpreted as follows. The melt composition is homogeneous prior to the primary phase nucleation. When the primary $\mathrm{Fe}_{2} \mathrm{Ti}$ phase nucleates and grows from the electrostatically levitated $\mathrm{Fe}_{59} \mathrm{Ti}_{41}$ alloy melt, the local alloy composition around growing primary $\mathrm{Fe}_{2} \mathrm{Ti}$ dendrites deviates greatly to FeTi phase side, as depicted in Figure 10(b3). In such a case, the solute segregation behavior contributes to satisfying composition condition of metastable coupled growth between $\mathrm{Fe}_{2} \mathrm{Ti}$ and FeTi phases. ${ }^{[14]}$ It may be why a little of eutectic-like microstructure forms within the interdendritic regions. 

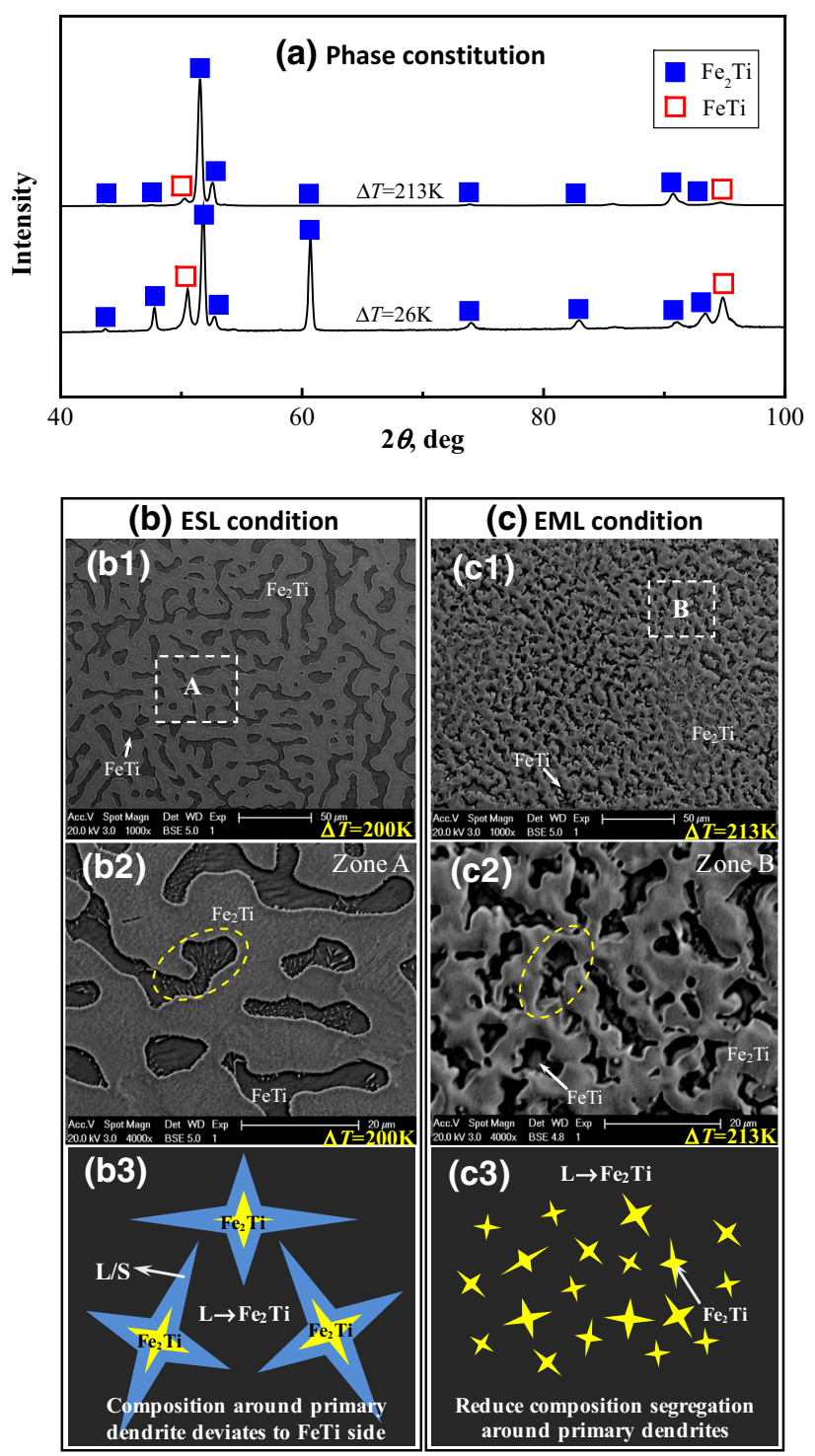

Fig. 10-A comparison of solidification microstructures and mechanisms for $\mathrm{Fe}_{59} \mathrm{Ti}_{41}$ alloy under electrostatic levitation (ESL) and electromagnetic levitation (EML) conditions: (a) phase constitution under EML condition, (bl) solidification microstructure of electrostatically levitated sample with $\Delta T=200 \mathrm{~K}$, (b2) the enlargement of solidification microstructure in (b1), (b3) the schematic diagram of solute redistribution at ESL, $(c 1)$ solidification microstructure of electromagnetically levitated sample with $\Delta T=213 \mathrm{~K},(c 2)$ the enlargement of solidification microstructure in (c1), (c3) the schematic diagram of solute redistribution at EML.

In contrast, due to the electromagnetic stirring, the solute segregation around these growing $\mathrm{Fe}_{2} \mathrm{Ti}$ dendrites is remarkably restrained in EML experiments, and the local composition in front of primary dendrites is close to the original composition. Therefore, the remaining liquid solidifies through the peritectic solidification mode, and finally forms a peritectic solidification microstructure under the EML condition.
4. Peritectic reaction difference between ESL and EML experiments

To quantitatively analyze the peritectic reaction difference between ESL and EML experiments, four physical parameters relating the peritectic reaction are defined from the cooling curves of electromagnetically levitated and electrostatically levitated $\mathrm{Fe}_{59} \mathrm{Ti}_{41}$ alloys. They are peritectic recalescence time $t_{\mathrm{pr}}$, peritectic recalescence degree $\Delta T_{\mathrm{pr}}$, peritectic recalescence rate $R_{\mathrm{pr}}$ and peritectic solidification time $t_{\mathrm{ps}}$, which have been designated in Figures 7(a) and (b). The $t_{\mathrm{pr}}$ corresponds to the time interval between $\mathrm{B}$ and $\mathrm{C}$ positions on the cooling curve in Figure 7, in which B position is the onset of peritectic reaction and $\mathrm{C}$ position locates at the highest temperature of peritectic recalescence peak. The $\Delta T_{\mathrm{pr}}\left(\Delta T_{\mathrm{pr}}=T_{\mathrm{C}}-T_{\mathrm{B}}\right)$ represents the temperature rising range due to the peritectic reaction, and the $R_{\mathrm{pr}}$ equals the ratio of $\Delta T_{\mathrm{pr}}$ and $t_{\mathrm{pr}}$. The $t_{\mathrm{ps}}$ is the time difference between $\mathrm{B}$ and $\mathrm{D}$ positions on the cooling curve in Figure 7, and B and D positions respectively denote the starting and finishing points of peritectic solidification. Understanding the effects of bulk undercooling $\Delta T$ and levitation condition on these parameters contributes to the advancement of peritectic solidification theory.

Figure 11(a) displays the measured peritectic recalescence time $t_{\mathrm{pr}}$ of levitated $\mathrm{Fe}_{59} \mathrm{Ti}_{41}$ alloy at various undercoolings. As $\Delta T$ rises, the formation of peritectic phase is easier, and the $t_{\mathrm{pr}}$ shows a decreasing trend. The variations of $t_{\mathrm{pr}}$ with $\Delta T$ of electrostatically and electromagnetically levitated $\mathrm{Fe}_{59} \mathrm{Ti}_{41}$ alloys respectively satisfy a power function and a linear function:

$$
\begin{gathered}
t_{\mathrm{pr}}=3.51 \Delta T^{-0.23} \text { at ESL } \\
t_{\mathrm{pr}}=1.02-9.25 \times 10^{-4} \Delta T \text { at EML }
\end{gathered}
$$

Evidently, as the undercooling rises, the value of $t_{\mathrm{pr}}$ decreases under the two conditions. At higher undercooling, primary solid phase is refined generally and it reacts more quickly with the remaining liquid to produce the peritectic phase. It explains the reason why $t_{\mathrm{pr}}$ is decreasing with the enlarged $\Delta T$. Comparing the $t_{\mathrm{pr}}$ at ESL and EML, it is apparent that the previous value is slightly larger than the latter one in the experimental undercooling range. Moreover, the difference in the two sets of data decreases gradually with the enhanced $\Delta T$. The possible interpretation is the EMS effect and higher cooling rates. The EMS accelerates the peritectic reaction proceeding, especially at slight undercoolings. This acceleration is reduced at highly undercooled state due to the rising melt viscosity and the weakened EMS effects. Besides, according to thermal balance, the high cooling rate contributes to shortening the peritectic recalescence time to some extent. Thus, the change of the peritectic recalescence time seems to be rather a combined effect of EMS and higher cooling rate in EML experiments. 


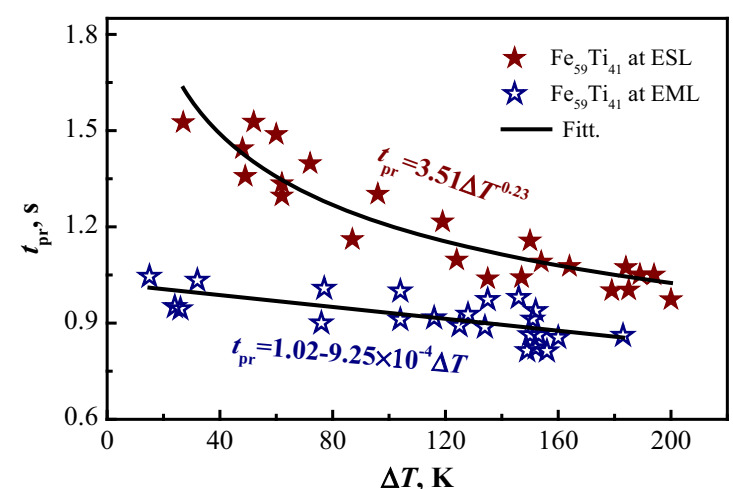

(a) $t_{\mathrm{pr}}$

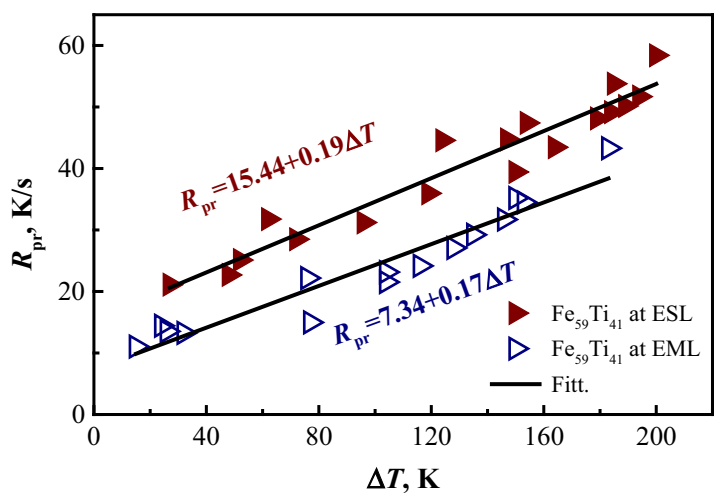

(c) $R_{\mathrm{pr}}$

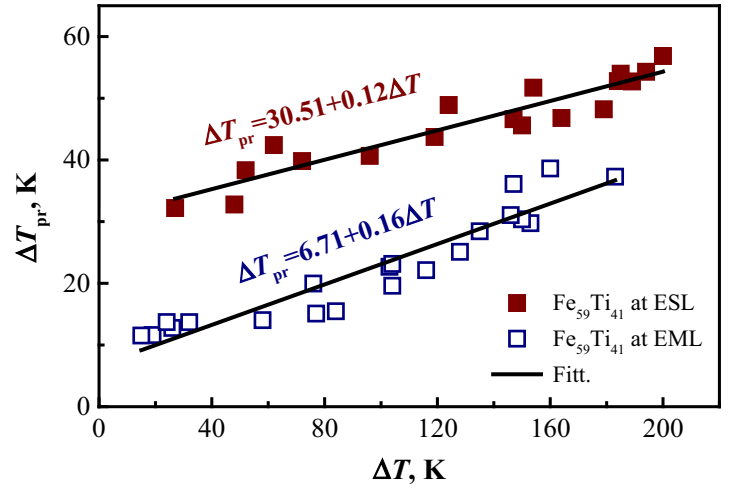

(b) $\Delta T_{\mathrm{pr}}$

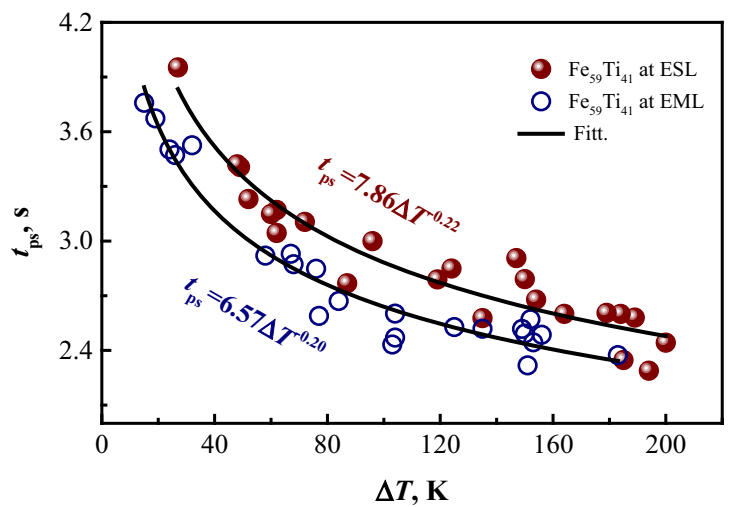

(d) $t_{\mathrm{ps}}$

Fig. 11-Effects of levitation condition and bulk undercooling on peritectic reaction kinetics for undercooled $\mathrm{Fe}_{59} \mathrm{Ti}_{41}$ alloys: $(a)$ peritectic recalescence time $t_{\mathrm{pr}},(b)$ peritectic recalescence degree $\Delta T_{\mathrm{pr}},(c)$ peritectic recalescence rate $R_{\mathrm{pr}},(d)$ peritectic solidification time $t_{\mathrm{ps}}$.

Figure 11(b) shows the determined peritectic recalescence degree $\Delta T_{\text {pr }}$ of levitated $\mathrm{Fe}_{59} \mathrm{Ti}_{41}$ alloy versus bulk undercooling and levitation condition. At both ESL and EML, a greater $\Delta T_{\mathrm{pr}}$ always occurs at a higher $\Delta T$. The undercooling dependences of peritectic recalescence degree $\Delta T_{\mathrm{pr}}$ for electrostatically levitated and electromagnetically levitated $\mathrm{Fe}_{59} \mathrm{Ti}_{41}$ alloys follow linear functions:

$$
\begin{aligned}
& \Delta T_{\mathrm{pr}}=30.51+0.12 \Delta T \text { at } \mathrm{ESL} \\
& \Delta T_{\mathrm{pr}}=6.71+0.16 \Delta T \text { at } \mathrm{EML}
\end{aligned}
$$

From Figure 7, it can be known that the sample heat dissipation of electromagnetically levitated alloys is more efficient because of their larger cooling rates. Thus, the heat dissipation with respect to the latent heat in the EML experiment is more than the ESL case. That is the reason why the peritectic recalescence degree $\Delta T_{\mathrm{pr}}$ of electrostatically levitated samples is greater at same undercoolings.

Using the data in Figures 11(a) and (b), peritectic recalescence rates $R_{\mathrm{pr}}$ are calculated and plotted in Figure 11(c). Clearly, the larger $\Delta T$ leads to a higher $R_{\mathrm{pr}}$, irrespective of experimental conditions. This means that peritectic reaction proceeds more intensively with the enlarged undercooling. The quantitative relationship between the $R_{\text {pr }}$ and $\Delta T$ for levitated $\mathrm{Fe}_{59} \mathrm{Ti}_{41}$ alloy can be well simulated by linear functions

$$
\begin{aligned}
& R_{\mathrm{pr}}=15.44+0.19 \Delta T \text { at } \mathrm{ESL} \\
& R_{\mathrm{pr}}=7.34+0.17 \Delta T \text { at EML }
\end{aligned}
$$

Apparently, the electromagnetically levitated $\mathrm{Fe}_{59} \mathrm{Ti}_{41}$ alloy has a slightly smaller $R_{\mathrm{pr}}$, which is ascribed to the combined effects of large cooling rates and additional electromagnetic stirring.

Figure 11(d) shows the undercooling dependence of peritectic solidification time $t_{\mathrm{ps}}$ in ESL and EML experiments. With the increase of $\Delta T, t_{\mathrm{ps}}$ displays a decreasing trend and their relation can be expressed as

$$
\begin{aligned}
& t_{\mathrm{ps}}=7.86 \Delta T^{-0.22} \text { at ESL } \\
& t_{\mathrm{ps}}=6.57 \Delta T^{-0.20} \text { at EML }
\end{aligned}
$$

Similar with $t_{\mathrm{pr}}$, the peritectic solidification time $t_{\mathrm{ps}}$ in the case of ESL is a little longer than that under EML condition. It further demonstrates that the EMS contributes to the proceeding of peritectic reaction and transformation. 


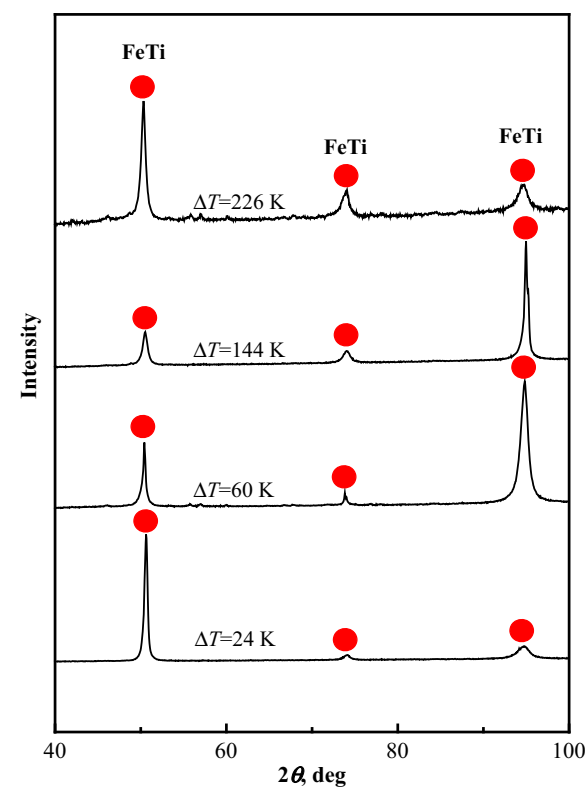

(a) Phase constitution

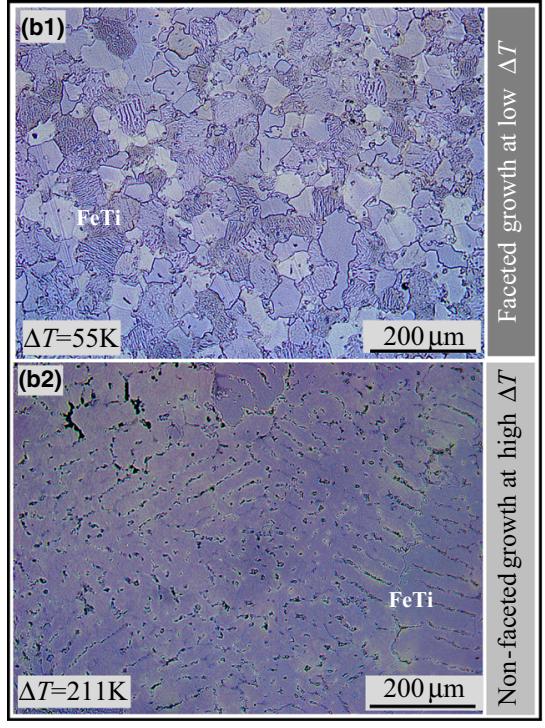

(b) Electrostatic levitation

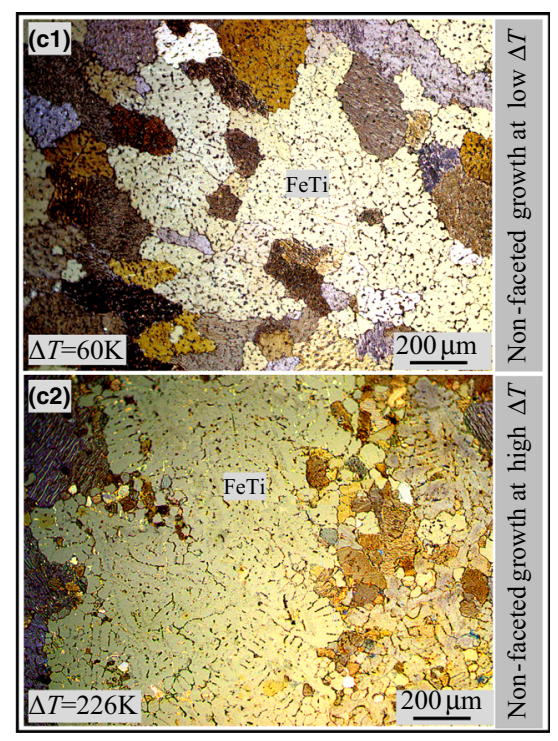

(c) Electromagnetic levitation

Fig. 12-A comparison of solidification microstructures for $\mathrm{Fe}_{50} \mathrm{Ti}_{50}$ alloy under electrostatic levitation and electromagnetic levitation conditions: (a) phase constitution at EML condition, $(b 1)$ and (b2) solidification microstructures at $\Delta T=55 \mathrm{~K}$ and $\Delta T=211 \mathrm{~K}$ in electrostatic levitation (ESL) experiments, $(c 1)$ and $(c 2)$ solidification microstructures at $\Delta T=60 \mathrm{~K}$ and $\Delta T=226 \mathrm{~K}$ in electromagnetic levitation (EML) experiments.

\section{Solidification Microstructure Distinction of $\mathrm{Fe}-\mathrm{Ti}$ Alloys Processed by ESL and EML Techniques}

To explore the EMS effects on the microstructure characteristics, solidification microstructures of electrostatically levitated and electromagnetically levitated $\mathrm{Fe}_{59} \mathrm{Ti}_{41}$ (or $\mathrm{Fe}_{50} \mathrm{Ti}_{50}$ ) alloys were comparatively analyzed in Figure 10 (or Figure 12). It can be concluded that the EMS has three aspects of effects on the solidification microstructure.

\section{a. Microstructure refinement caused by grain fragmenta-} tion and multiplication As illustrated in Figures 10(b1), (c1), 12(b2) and (c2), the solidification microstructures of electromagnetically levitated $\mathrm{Fe}_{59} \mathrm{Ti}_{41}$ and $\mathrm{Fe}_{50} \mathrm{Ti}_{50}$ alloys are significantly refined in contrast with the microstructures obtained at ESL. Furthermore, this refinement occurs irrespective of phase constitutions. Under the EML condition, the EMS force inside the levitated alloy melt always induces melt flow that can usually lead to forced convection. So far, it has been widely reported that the forced convection caused by EMS can improve grain fragmentation and thus promote grain multiplication to produce the refined microstructure.

b. Solute redistribution induces the variation of microstructure type In the experimental undercooling range, hypoperitectic $\mathrm{Fe}_{59} \mathrm{Ti}_{41}$ alloy forms a peritectic solidification microstructure under the effects of EMS, as shown in Figure 10(c2). However, in the absence of EMS, it develops hypoeutectic-like (or hyperperitectic-like) microstructures as seen in Figure 10(b2). Evidently, the EMS changes the solidification microstructure type for the $\mathrm{Fe}_{59} \mathrm{Ti}_{41}$ alloy. During the growth of primary $\mathrm{Fe}_{2} \mathrm{Ti}$ phase, the solute composition around the primary phase greatly deviates to FeTi phase side as the EMS is absent, which contributes to satisfying the composition condition for the metastable coupled growth of primary and peritectic phases. Accordingly, an eutectic-type microstructure is preserved in ESL experiments. If the EMS is imposed, the solute composition around the primary phase will be very close to the original composition and the peritectic-type solidification microstructure is easy to be formed in EML experiments.

c. Faceted-growth morphology of intermetallic compound phases disappears due to the fluid scouring and fluid erosion At small $\Delta T$, the solidification microstructure of electrostatically levitated $\mathrm{Fe}_{50} \mathrm{Ti}_{50}$ alloy only consists of intermetallic compound FeTi phase and it has an evident faceted-growth feature as shown in Figure 12(b1). In contrast, the faceted-growth morphology is not observed for electromagnetically levitated sample with almost equal undercoolings, as displayed in Figure 12(c1). During the direct nucleation and growth of FeTi phase from the undercooled $\mathrm{Fe}_{50} \mathrm{Ti}_{50}$ alloy melt, the EMS existence at EML brings serious fluid scouring and fluid erosion effects in the alloy melt. The edges and corners of the faceted-growth morphology may be polished as a result of the mechanical collision, fluid scouring and fluid erosion, and the faceted-growth morphology is finally changed into a cluster-like morphology. As the undercooling level is improved, the intermetallic compound FeTi phase does not solidify as usual. In this case, it solidifies like a solid solution phase and the final solidification microstructures under ESL and EML condition appear as dendritic morphologies. 


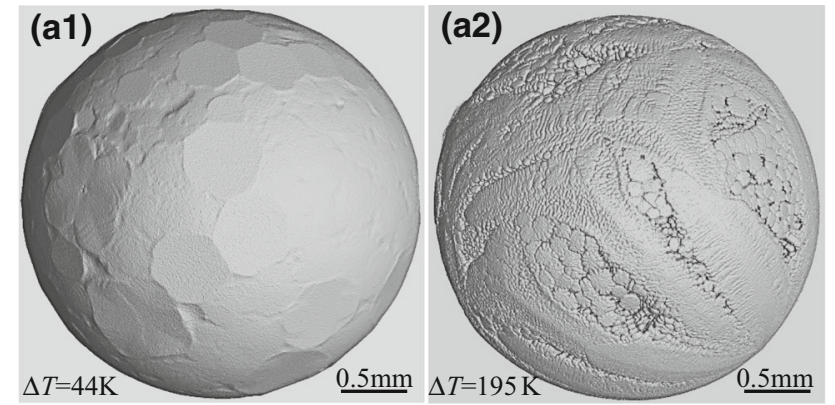

(a) Surface morphologies under ESL condition

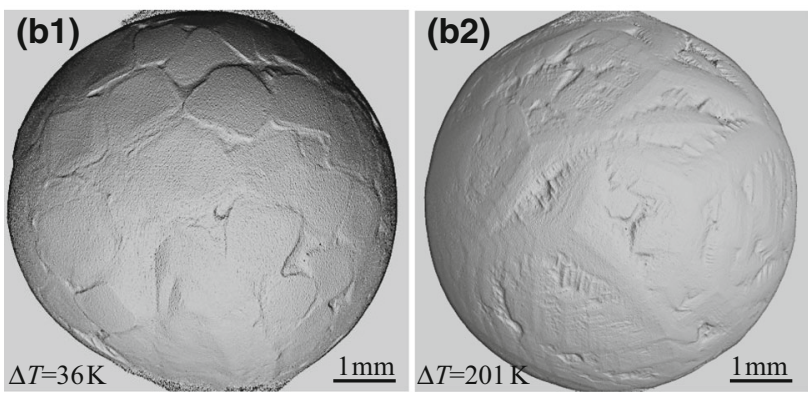

(b) Surface morphologies under EML condition

Fig. 13-3D X-ray computed tomography for surface morphologies of electrostatically levitated and electromagnetically levitated $\mathrm{Fe}_{50} \mathrm{Ti}_{50}$ alloys: (al) and (a2) surface morphologies of electrostatically levitated samples with $44 \mathrm{~K}$ and $195 \mathrm{~K}$ undercooling, respectively, and (b1) and (b2) surface morphologies of electromagnetically levitated samples with 36 K and $201 \mathrm{~K}$ undercooling, respectively.

To further demonstrate the EMS effect and the undercooling effect, surface morphologies of electrostatically levitated and electromagnetically levitated $\mathrm{Fe}_{50} \mathrm{Ti}_{50}$ alloys were characterized by 3D X-ray computed tomography technique, as given in Figure 13. For the EML sample with a small undercooling, the faceted-growth characteristics of sample upper part close to the control coil is more evident than other regions, as illustrated in Figure 13(b1). This agrees well with our calculations about fluid flow dynamics in Figure 4. The fluid velocity of sample upper part is remarkably less than other regions. The faceted-growth mode will be favored if the forced convection induced by EMS is very weak. If we further compare with the ESL sample with slight undercoolings, it is confirmed that the EMS changes the faceted-growth mode of intermetallic compounds. At highly undercooled state, both the surface morphologies in ESL and EML experiments evolve into a dendritic morphology and this is also consistent with internal microstructure evolution.

\section{CONCLUSIONS}

In the present work, the effects of electromagnetic stirring (EMS) on the peritectic solidification kinetics for containerlessly processed liquid $\mathrm{Fe}-\mathrm{Ti}$ alloys were analyzed by a proposed physical model and confirmed by a comparative study of ESL and EML. The main conclusions are given as follows.

(1) Dendrite growth effect: The EMS alters the transport of the solute rejected at the liquid/solid interface and affects dendrite growth velocity. The undercooling dependence of primary dendrite growth velocity $V$ for levitated liquid Fe-Ti alloys satisfies power functions. The measured $V$ of $\mathrm{Fe}_{50} \mathrm{Ti}_{50}$ alloy at EML is slightly larger than that at ESL if $\Delta T<150 \mathrm{~K}$. Once the $\Delta T$ exceeds this threshold value, the growth velocity data under the two conditions become consistent.

(2) Concentration field effect: The EMS facilitates mass transfer and homogenizes the concentration field. During the primary $\mathrm{Fe}_{2} \mathrm{Ti}$ dendrite growth, the solute concentration $C_{\mathrm{L}}^{*}$ around growing $\mathrm{Fe}_{2} \mathrm{Ti}$ dendrites of an electrostatically levitated $\mathrm{Fe}_{59} \mathrm{Ti}_{41}$ alloy deviates toward FeTi phase side. However, as the additional EMS facilitates the solute transfer at EML, the $C_{\mathrm{L}}^{*}$ around $\mathrm{Fe}_{2} \mathrm{Ti}$ dendrites is close to the original composition. The concentration field effect has been indirectly demonstrated by the solidification microstructures at ESL and EML.

(3) Peritectic reaction effect: The EMS induces primary grain refinement, expedites solute diffusion and accelerates the peritectic reaction. As $\Delta T$ rises for $\mathrm{Fe}_{59} \mathrm{Ti}_{41}$ alloy, the peritectic recalescence degree $\Delta T_{\mathrm{pr}}$ and the peritectic recalescence rate $R_{\mathrm{pr}}$ increase linearly, while the peritectic recalescence time $t_{\mathrm{pr}}$ and the peritectic solidification time $t_{\mathrm{ps}}$ decrease gradually. In contrast with ESL results, both the $t_{\mathrm{pr}}$ and $t_{\mathrm{ps}}$ are a little smaller, and it reveals that the EMS expedites the peritectic reaction.

(4) Microstructure evolution effect: The EMS promotes microstructure refinement, modulates microstructure type and changes faceted-growth mode. The microstructure distinctions of $\mathrm{Fe}-\mathrm{Ti}$ alloys processed by ESL and EML show that the EMS induces the microstructure refinement through the grain fragmentation and multiplication. Moreover, hypoperitectic $\mathrm{Fe}_{59} \mathrm{Ti}_{41}$ alloy forms a peritectic solidification microstructure in the presence of EMS, whereas it develops a hypoeutectic-like (hypereutectic-like) microstructure in the absence of EMS. Besides, except for the high undercoolings, the EMS also alters the faceted-growth mode of intermetallic compound phases.

\section{ACKNOWLEDGMENTS}

The authors are very grateful to Dr. W.L. Wang, Dr. S.J. Yang, and Mr. P.F. Zou for their help with EML and ESL experiments. The authors would also like to thank Dr. I. Kaban and M. Sc. A. Andreoli for 
their help with the 3D X-ray computed tomography characterization. This work is financially supported by National Natural Science Foundation of China (Grant Nos. 51327901 and 51771154), Key Research and Development Program of Shaanxi (Program No.2019GY-152), and 2018 Sino-German (CSC-DAAD) Postdoc Scholarship Program.

\section{ELECTRONIC SUPPLEMENTARY MATERIAL}

The online version of this article (doi:https://doi.org/ 10.1007/s11661-020-05745-w) contains supplementary material, which is available to authorized users.

\section{REFERENCES}

1. F.P. Dai, Y.H. Wu, W.L. Wang, and B. Wei: Metall. Mater. Trans. A, 2018, vol. 49A, pp. 5478-87.

2. W.J. Boettinger, D.E. Newbury, N.W.M. Ritchie, M.E. Williams, U.R. Kattner, E.A. Lass, K.-W. Moon, M.B. Katz, and J.H. Perepezko: Metall. Mater. Trans. A, 2019, vol. 50A, pp. $772-88$.

3. J. Valloton, J.A. Dantzig, M. Plapp, and M. Rappaz: Acta Mater., 2013, vol. 61, pp. 5549-60.

4. J.E. Rodriguez and D.M. Matson: Acta Mater., 2017, vol. 129, pp. $408-14$.

5. P. Peng, X.Z. Li, Y.Q. Su, and J.J. Guo: Appl. Phys. Lett., 2016, vol. 109 , art. no. 021603.

6. M. Leonhardt, W. Löser, and H.G. Lindenkreuz: Acta Mater., 2002, vol. 50, pp. 725-34.

7. P. Lü, H.P. Wang, and B. Wei: Metall. Mater. Trans. A, 2019, vol. 50A, pp. 789-803

8. M. Asta, C. Beckermann, A. Karma, W. Kurz, R. Napolitano, M. Plapp, G. Purdy, M. Rappaz, and R. Trivedi: Acta Mater., 2009, vol. 57, pp. 941-71.

9. D.A. Basha, N. Ravishankar, and K. Chattopadhyay: Scripta Mater., 2018, vol. 143, pp. 68-71.

10. A. Jacot, M. Sumida, and W. Kurz: Acta Mater., 2011, vol. 59, pp. $1716-24$.

11. S. Abraham, R. Bodnar, J. Lonnqvist, F. Shahbazian, A. Lagerstedt, and M. Andersson: Metall. Mater. Trans. A, 2019, vol. 50A, pp. 2259-71.

12. D.M. Liu, X.Z. Li, Y.Q. Su, P. Peng, L.S. Luo, J.J. Guo, and H.Z. Fu: Acta Mater., 2012, vol. 60, pp. 2679-88.

13. A. Ludwig and J. Mogeritsch: J. Cryst. Growth, 2016, vol. 455, pp. $99-104$.

14. Y.H. Wu, J. Chang, W.L. Wang, and B. Wei: Appl. Phys. Lett., 2016, vol. 109, art. no. 154101 .
15. C.J. Todaro, M.A. Easton, D. Qiu, G. Wang, D.H. Stjohn, and M. Qian: Metall. Mater. Trans. A, 2017, vol. 48A, pp. 557990.

16. S. Akamatsu and M. Plapp: Curr. Opin. Solid St. M., 2016, vol. 20, pp. 46-54.

17. S.Y. Pan, M.F. Zhu, and M. Rettenmayr: Acta Mater., 2017, vol. 132 , pp. $565-75$.

18. S. Jeon, D.-H. Kang, Y.H. Lee, S. Lee, and G.W. Lee: J. Chem. Phys., 2016, vol. 145, art. no. 174504

19. D.G. Quirinale, G.E. Rustan, A. Kreyssig, and A.I. Goldman: Appl. Phys. Lett., 2015, vol. 106, art. no. 241906.

20. M.L. Johnson, P.C. Gibbons, A.J. Vogt, and K.F. Kelton: $J$. Alloy. Compd., 2017, vol. 725, pp. 1217-22.

21. S.B. Luo, W.L. Wang, J. Chang, Z.C. Xia, and B. Wei: Acta Mater., 2014, vol. 69, pp. 355-64.

22. B. Bochtler, O. Gross, I. Gallino, and R. Busch: Acta Mater., 2016, vol. 118, pp. 129-39.

23. S.J. McCormack, R.J. Weber, and W.M. Kriven: Acta Mater., 2018, vol. 161, pp. 127-37.

24. L. Cox, A. Croxford, B.W. Drinkwater, and A. Marzo: Appl. Phys. Lett., 2018, vol. 113, art. no. 054101.

25. S.Y. Park and W.J. Kim: Metall. Mater. Trans. A, 2017, vol. 48, pp. 3523-39.

26. T. Yuan, S. Kou, and Z. Luo: Acta Mater., 2016, vol. 106, pp. 144-54.

27. E. Liotti, A. Lui, R. Vincent, S. Kumar, Z. Guo, T. Connolley, I.P. Dolbnya, M. Hart, L. Arnberg, R.H. Mathiesen, and P.S. Grant: Acta Mater., 2014, vol. 70, pp. 228-39.

28. S. Agrawal, A.K. Ghose, and I. Chakrabarty: Mater. Design, 2017, vol. 113, pp. 195-206.

29. I. Kaldre, Y. Fautrelle, J. Etay, A. Bojarevics, and L. Buligins: $J$. Cryst. Growth, 2014, vol. 402, pp. 230-33.

30. Y.H. Zhang, Y.Y. Xu, C.Y. Ye, C. Sheng, J. Sun, G. Wang, X.C. Miao, C.J. Song, and Q.J. Zhai: Sci. Rep., 2018, vol. 8, art. no. 3242 .

31. M. Vynnycky: Metall. Mater. Trans. B, 2018, vol. 49B, pp. $399-410$

32. F. Lin and W.Y. Shi: Int. J. Heat Mass Tran., 2018, vol. 122, pp. 69-77.

33. S. Spitans, E. Baake, B. Nacke, and A. Jakovics: Metall. Mater. Trans. B, 2016, vol. 47B, pp. 522-36.

34. A. Kermanpur, M. Jafari, and M. Vaghayenegar: J. Mater. Process Tech., 2011, vol. 211, pp. 222-29.

35. X. Cai, H.P. Wang, P. Lü, and B. Wei: Metall. Mater. Trans. B, 2018, vol. 49B, pp. 2252-60.

36. F. Lin and W.Y. Shi: Metall. Mater. Trans. B, 2015, vol. 46B, pp. 1895-901.

37. N. Shevchenko, O. Roshchupkina, O. Sokolova, and S. Eckert: $J$. Cryst. Growth, 2015, vol. 417, pp. 1-8.

38. D.K. Sun, M.F. Zhu, S.Y. Pan, and D. Raabe: Acta Mater., 2009, vol. 57, pp. 1755-67.

39. C.J. Smithells: Metals Reference Book, 6th ed., Butterworth, London, 1984

Publisher's Note Springer Nature remains neutral with regard to jurisdictional claims in published maps and institutional affiliations. 